\title{
The amphibians and reptiles of Manu National Park and its buffer zone, Amazon basin and eastern slopes of the Andes, Peru
}

\author{
Alessandro Catenazzi ${ }^{1,4}$, Edgar Lehr ${ }^{2}$ \& Rudolf von May ${ }^{3}$ \\ ${ }^{1}$ Department of Zoology, Southern Illinois University Carbondale - SIU, Carbondale, IL 62901, USA \\ ${ }^{2}$ Department of Biology, Illinois Wesleyan University - IWU, Bloomington, IL 61701, USA \\ ${ }^{3}$ Museum of Vertebrate Zoology, University of California - UC, Berkeley, CA 94720, USA \\ ${ }^{4}$ Corresponding author: Alessandro Catenazzi, e-mail: acatenazzi@gmail.com
}

CATENAZZI, A., LEHR, E. \& VON MAY, R. The amphibians and reptiles of Manu National Park and its buffer zone, Amazon basin and eastern slopes of the Andes, Peru. Biota Neotrop. 13(4): http://www. biotaneotropica.org.br/v13n4/en/abstract?inventory+bn02813042013

\begin{abstract}
We compile a list of all amphibians and reptiles known to occur within Manu National Park, Peru and its buffer zone, located in one of the world's biodiversity hotspots. Covering approximately $0.01 \%$ of the planet's terrestrial surface, this protected area preserves 155 species of amphibians and 132 species of reptiles, corresponding to $2.2 \%$ and $1.5 \%$ respectively of the known diversity for these groups. Moreover, Manu National Park preserves natural habitats and populations of one critically endangered (Atelopus erythropus), three endangered (Bryophryne cophites, Pristimantis cosnipatae and Psychrophrynella usurpator), three vulnerable amphibians (Atelopus tricolor, Gastrotheca excubitor, Rhinella manu) and two vulnerable reptiles (Chelonoidis denticulata, Podocnemis unifilis), according to the threat categories of the IUCN Red List.

Keywords: herpetofauna, biodiversity hotspots, Cusco Region, Madre de Dios Region, Amazonia.
\end{abstract}

CATENAZZI, A., LEHR, E. \& VON MAY, R. Los anfibios y reptiles del Parque Nacional del Manu y su área de amortiguamiento, cuenca Amazónica y vertientes orientales de los Andes, Perú. Biota Neotrop. 13(4): http://www.biotaneotropica.org.br/v13n4/pt/abstract?inventory+bn02813042013

Resumen: Producimos una lista de las especies de anfibios y reptiles conocidas del Parque Nacional del Manu, Perú y su zona de amortiguamiento, ubicados en unas de las regiones con más biodiversidad en el planeta. El área protegida cubre tan solo cerca del $0.01 \%$ de la superficie terrestre mundial, sin embargo conserva a 155 especies de anfibios y 132 especies de reptiles, lo cual corresponde al 2.2\% de todos los anfibios y al 1.5\% de todos los reptiles conocidos. Además, el Parque Nacional del Manu protege los hábitats y las poblaciones de una especie críticamente amenazada (Atelopus erythropus), tres especies amenazadas (Bryophryne cophites, Pristimantis cosnipatae y Psychrophrynella usurpator), tres especies vulnerables de anfibios (Atelopus tricolor, Gastrotheca excubitor, Rhinella manu), y dos especies vulnerables de reptiles (Chelonoidis denticulata, Podocnemis unifilis), de acuerdo a las categorías de amenaza de la Lista Roja de la UICN.

Palabras clave: herpetofauna, biodiversidad, Cusco, Madre de Dios, Amazonía. 


\section{Introduction}

The eastern slopes of the Andes have one of the highest diversity of amphibians in the world (Duellman 1999, Stuart et al. 2004), as well as high diversity of reptiles (Duellman 2005, von May et al. 2009). Peru is a mega-diverse country hosting approximately 571 species of amphibians (Frost 2013) and 437 species of reptiles (Uetz \& Hošek 2013). The most important biodiversity hotspot for amphibians, the Tropical Andes (Myers et al. 2000), runs from north to south along the western half of Peru's territory. Several protected areas have been created in the Peruvian Andes over the past three decades (SERNANP 2010). However, our knowledge of the biodiversity within these areas is very fragmentary.

For example in southeastern Peru, 10 nationally protected areas extend from the Amazon lowlands in the Department of
Madre de Dios to the foothill of the Andes of Cusco and Puno (Figure 1), yet none of these areas has a comprehensive list of amphibian and reptiles species. One of such areas, Manu National Park (Manu NP), is unique in covering the entire watershed of the Manu river, a large tributary to the Alto Madre de Dios river (the upper Madre de Dios river, a tributary to the Madeira river). In the lowland floodplain and terra firme forests along the Manu River, inside Manu NP, several publications reported preliminary lists of amphibians and reptiles known to occur at two sites, Cocha Cashu Biological Station (Rodríguez 1987, 1992, Rodríguez \& Cadle 1990) and Pakitza (Morales \& McDiarmid 1996). Recent studies have produced extensive lists for Los Amigos Conservation Concession (von May et al. 2006, 2009, 2010). Los Amigos lies outside of Manu NP, but its northern edge overlaps with the park's buffer zone. Most of our knowledge about amphibian and reptilian diversity in the

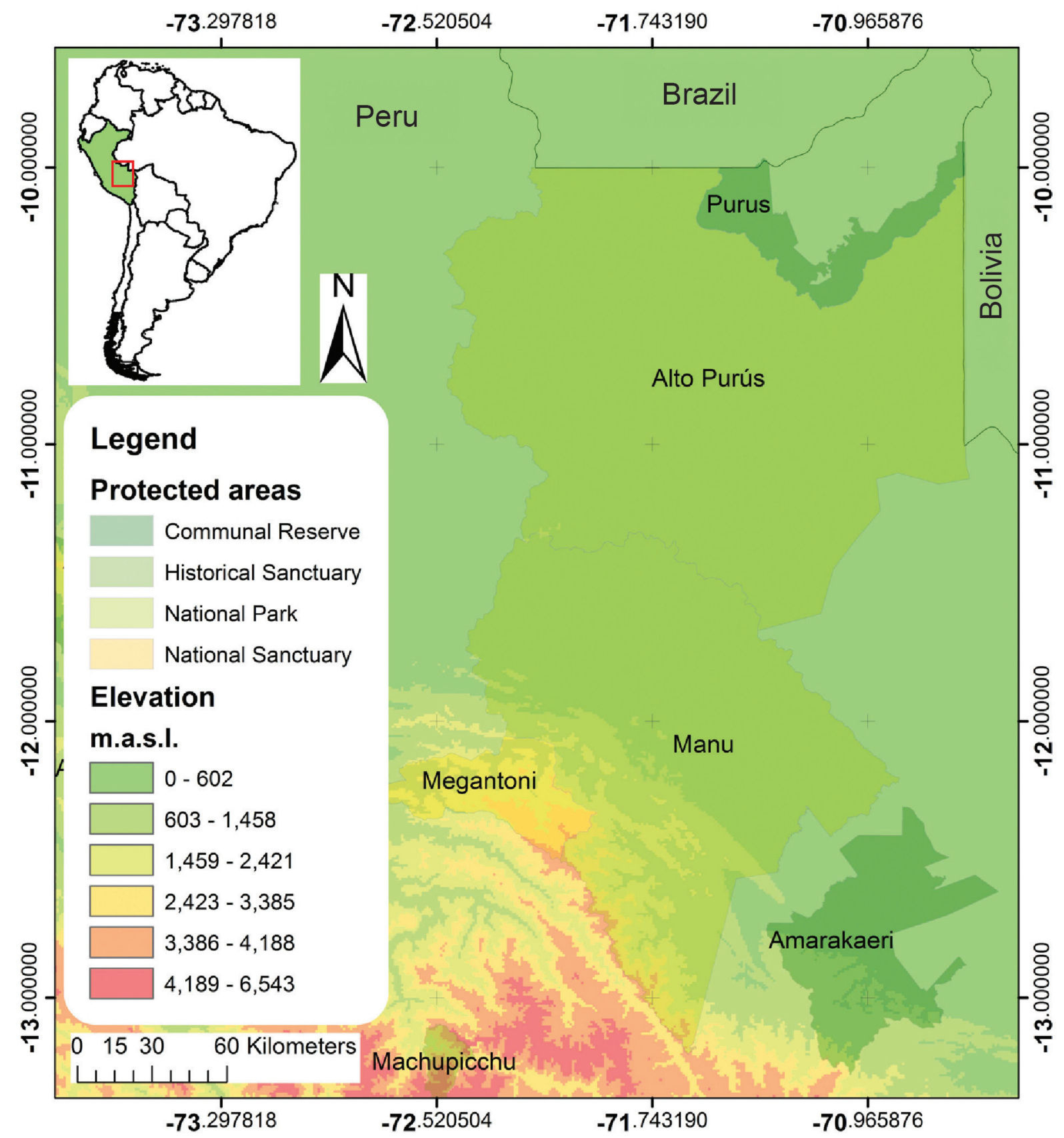

Figure 1. Map of Manu National Park and adjacent protected areas in southeastern Peru. The inset map shows the location of Peru in South America. 
Andean foothills comes from studies conducted in the Kosñipata valley (Hurtado \& Blanco 1994, Catenazzi \& Rodriguez 2001, Catenazzi et al. 2009, 2011, 2012, Lehr \& Catenazzi 2008, 2009a, b, unpublished field data), including Villa Carmen and Wayqecha Biological Stations. The upper Kosñipata valley (above 2500-3000 $\mathrm{m}$ ) is part of Manu NP, but the rest of the valley between Pillahuata $(2500 \mathrm{~m})$ and Pillcopata $(600 \mathrm{~m})$, along the Paucartambo-Shintuya road, is part of the park's buffer zone.

Despite these studies and preliminary inventories, Manu NP still lacks a comprehensive list of amphibian and reptile taxa. The lack of species lists hinders conservation and management efforts. The upper part of Manu NP is currently experiencing a collapse in amphibian species richness and abundance following the spread of the highly pathogenic fungus Batrachochytrium dendrobatidis (Catenazzi et al. 2011). A better knowledge of amphibian diversity and distribution will assist monitoring and mitigation efforts. Therefore, the aim of this paper is to gather data from previous lists, species descriptions, museum records and our own fieldwork in the region to compile a checklist of all amphibians and reptiles that have been found within Manu National Park and its adjacent buffer zone.

\section{Methods}

Manu National Park (Manu NP) is located in southeastern Peru (Regions of Cusco and Madre de Dios) and covers $17163 \mathrm{~km}^{2}$ of Amazonian lowland, submontane, montane and high-elevation Andean habitats between $150 \mathrm{~m}$ and $4200 \mathrm{~m}$ elevation (Figure 1). The park protects the entire watershed of the Manu River in the upper Madre de Dios basin. Manu NP was established in 1973, recognized as a Reserve of Biosphere by the UNESCO in 1977, and pronounced a World Heritage Site in 1987; it is Peru's second largest national park and it lies in one of the world's biodiversity hotspots (Myers et al. 2000).

Occurrence of taxa was determined on the basis of the original species descriptions, listings in species descriptions of similar or sympatric taxa, museum records and/or our own fieldwork in the region (Catenazzi \& Rodriguez 2001, Duellman \& Lehr 2009, von May 2009, von May et al. 2009, 2010, von May \& Donnelly 2009, Catenazzi et al. 2011). Some species have not been reported from Manu NP or its buffer zone, but occur both north and south of the area, such that their presence in Manu NP is very likely. These species are (in parenthesis references in support of the geographic distribution of each species): Rhinella leptoscelis (Padial et al. 2009), Hyalinobatrachium carlesvilai (Castroviejo-Fisher et al. 2009), Dendropsophus bokermanni and D. rossalleni (Duellman 2005), Apostolepis nigroterminata (Harvey 1999), Xenoxybelis boulengeri (Duellman 2005), Xenodon rabdocephalus (Duellman 2005), Micrurus narduccii (Campbell \& Lamar 1989), Bothriopsis oligolepis and B. taeniata (Campbell \& Lamar 1989), Bothrocophias microphthalmus (Gutberlet \& Campbell 2001), Paleosuchus palpebrosus (Duellman 2005). We have also listed 8 frog and one squamate species which are still not described, but known to represent new species.

General terrestrial habitat categories follow the references within the squared brackets. For the lowlands (300-500 m), we use forest types that are widely recognized by plant and animal ecologists working in western Amazonia (Pitman et al. 1999, Larsen et al. 2006, Griscom et al. 2007). One of these forest types, the floodplain, can be classified in two general categories: mature floodplain forest and early successional floodplain forest. However, because species recorded in the early successional forest can also be found in the mature floodplain forest (von May et al. 2010), we simply refer to floodplain forest as the major habitat category representing these two floodplain forest types. If a species was primarily associated with a permanent aquatic habitat such as a lake or a river, we referred to that particular aquatic habitat. In contrast, if a species was temporarily associated with smaller aquatic habitats such as temporary ponds or creeks embedded in major terrestrial habitats, we referred to that terrestrial habitat. We did not differentiate between forest types in the Andean piedmont (500-1000 m), where we used the general category of sub-montane rainforest. For both the lowlands and the foothills of the Andes, we recognized Mauritia flexuosa swamp and bamboo (Guadua spp.) forest as distinct habitats. The cloud forest category refers to all montane forests between 1000-3600 m; additional highelevation habitat types were the high-Andean grasslands or puna, dominated by Stipa ichu, and the montane scrub, which represents elfin forests and xeric vegetation growing on exposed slopes or ridges.

For taxonomy we followed Blackburn \& Wake (2011) for amphibians, Castoe et al. (2004) for gymnophthalmid lizards, Le et al. (2006) for tortoises and McCord et al. (2001) for chelid turtles.

\section{Results and Discussion}

We list 155 species of amphibians (Table 1) and 132 species of reptiles (Table 2) currently known to occur or suspected to occur within Manu NP. The cumulative number of species descriptions by year differs between amphibians and reptiles (Figure 2), because for reptiles the curve reached a number close to the asymptote in the decade of 1960-1970, whereas for amphibians this very same decade corresponded to a sharp increase in the number of new species described for Manu NP. The construction of the PaucartamboShintuya road in the late 60 s greatly facilitated access to highelevation and montane habitats. William E. Duellman and associates collected along this road from 1971-1975, and subsequently described several new anuran species from this region (Duellman 1976, 1978). Although the cumulative number of amphibians already exceeded the number of reptiles during the decade of 1990-2000, the pattern of accelerated discoveries of new and often endemic amphibian species is still holding up during the current decade (Catenazzi et al. 2012, Chaparro et al. 2007, De la Riva \& Chaparro 2005, De la Riva et al. 2008, Duellman et al. 2011, Padial et al. 2007, Lehr \& Catenazzi 2008, 2009a,b, Lehr \& von May 2009, Padial et al. 2012), and we are confident that several more species will be added to this list over the next few years. We expect that the final number of amphibian species will be greater than the number of reptile species, because the diversity of squamates decreases with elevation at a much faster rate than the diversity of anurans (Navas 2003). Squamates and anurans constitute the bulk of respectively reptilian and amphibian diversity. Given the wide elevational gradient and large area of montane and high-elevation habitats protected by Manu NP, the greater relative richness of anurans vs. squamates is probably replicated across several watersheds (and not just the Kosñipata watershed), further contributing to the amphibian primacy in species richness.

\section{Conservation Remarks}

Manu NP preserves natural habitats and populations of one critically endangered (Atelopus erythropus), three endangered (Bryophryne cophites, Pristimantis cosnipatae and Psychrophrynella usurpator), three vulnerable amphibians (Atelopus tricolor, Gastrotheca excubitor, Rhinella manu; von May et al. 2008) and two vulnerable reptiles (Chelonoidis denticulata, Podocnemis unifilis; Figure 3), based on the IUCN Red List of Threatened Species (http:// www.iucnredlist.org/). It is a place of exceptional biological diversity which currently stands as the protected area with the highest number of species of amphibians and reptiles on the planet: three cecilian, one salamander, 150 anuran, 120 squamate, four crocodilian and eight 
Catenazzi, A. et al.

Table 1. Amphibians of Manu National Park, Peru. The asterisk indicates species whose type locality is within Manu NP or its buffer zone. Species new to science but not yet described are listed as "sp." Abbreviations for habitat types are: for lowland forest $(300-500 \mathrm{~m})$ : $\mathrm{FP}=$ floodplain forest, $\mathrm{TF}=$ tierra firme forest, $\mathrm{SW}=$ Mauritia flexuosa swamp (aguajal), BF = bamboo (Guadua spp.) forest (pacal); for lowland aquatic or semi-aquatic habitat (300-500 m): LA=lake shore or floating vegetation around lake, $\mathrm{RI}=$ river bank or sandbar or pebble beach; for the piedmont of the Andes $(500-1000 \mathrm{~m})$ : $\mathrm{SM}=$ submontane rainforest, $\mathrm{SW}=$ Mauritia flexuosa swamp (aguajal), $\mathrm{BF}=$ bamboo (Guadua spp.) forest (pacal); for montane and high-Andean habitats $(1000-4000 \mathrm{~m})$ : $\mathrm{CF}=\mathrm{cloud}$ forest, $\mathrm{MS}=$ montane scrub, $\mathrm{PU}=$ puna (high-Andean grasslands).

\begin{tabular}{ccccc}
\hline Family & Species & \multicolumn{4}{c}{ Habitat type } \\
\hline & & FP & TF & LA RI BF SW SM CF MS PU \\
\hline
\end{tabular}

Order Anura

AROMOBATIDAE *Allobates alessandroi (Grant \& Rodríguez, 2001)

*Allobates conspicuus (Morales, 2002)

Allobates femoralis (Boulenger, 1884)

Allobates trilineatus (Boulenger, 1884 "1883")

BUFONIDAE

Atelopus erythropus Boulenger, 1903

Atelopus tricolor Boulenger, 1902

Amazophrynella minuta (Melin, 1941)

Rhaebo guttatus (Schneider, 1799)

Rhinella inca (Stejneger, 1913)

Rhinella leptoscelis (Boulenger, 1912)

*Rhinella manu Chaparro, Pramuk \& Gluesenkamp, 2007

Rhinella margaritifera (Laurenti, 1768)

Rhinella marina (Linnaeus, 1758)

Rhinella poeppigii (Tschudi, 1845)

Rhinella veraguensis (Schmidt, 1857)

CENTROLENIDAE Centrolene sabini Catenazzi, von May, Lehr, GagliardiUrrutia \& Guayasamin, 2012

Hyalinobatrachium bergeri (Cannatella, 1980)

Hyalinobatrachium carlesvilai Castroviejo-Fisher, Padial, Chaparro, Aguayo-Vedia, and De la Riva, 2009

Nymphargus pluvialis (Cannatella \& Duellman, 1982)

*Nymphargus truebae (Duellman, 1976)

*Rulyrana spiculata (Duellman, 1976)

Teratohyla midas (Lynch \& Duellman, 1973)

$\begin{array}{ll}\text { CERATOPHRYIDAE } & \text { Ceratophrys cornuta (Linnaeus, 1758) } \\ & * \text { Telmatobius mendelsoni De la Riva, Trueb \& Duellman, }\end{array}$ 2012

Telmatobius timens De la Riva, Aparicio \& Ríos, 2005

DENDROBATIDAE Ameerega hahneli (Boulenger, 1884)

*Ameerega macero (Rodríguez \& Myers, 1993)

Ameerega picta (Bibron in Tschudi, 1838)

Ameerega simulans (Myers, Rodríguez \& Icochea, 1998)

Ameerega trivittata (Spix, 1824)

Ranitomeya sirensis Aichinger 1991

Ranitomeya uakarii Brown et al. 2006

*Gastrotheca antoniiochoai (De la Riva \& Chaparro, 2005)

*Gastrotheca excubitor Duellman \& Fritts, 1972

Gastrotheca marsupiata (Dumeril \& Bibron, 1841)

*Gastrotheca nebulanastes Duellman, Catenazzi \& Blackburn, 2011

Gastrotheca testudinea (Jiménez de la Espada, 1870)

*Hemiphractus helioi Sheil \& Mendelson, 2001

Hemiphractus scutatus (Spix, 1824)

HYLIDAE Cruziohyla craspedopus (Funkhouser, 1957)

Dendropsophus acreanus (Bokermann, 1964)

Dendropsophus allenorum (Duellman \& Trueb, 1989)

Dendropsophus bifurcus (Andersson, 1945)

Dendropsophus bokermanni (Goin, 1960)
X X

X X

$\mathrm{X} \quad \mathrm{X}$

X X

X

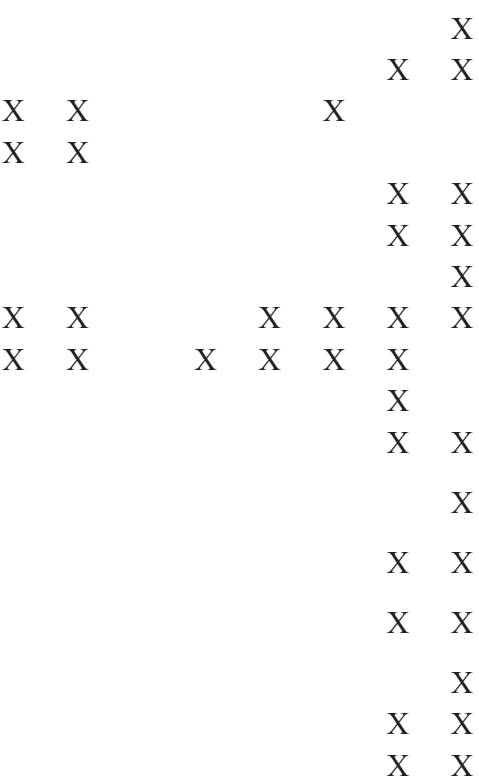

$\mathrm{X} \quad \mathrm{X}$

$\mathrm{X} \quad \mathrm{X}$

X

X

X X

X X

$\mathrm{X}$

$\mathrm{X} \quad \mathrm{X}$

$\mathrm{X} \quad \mathrm{X}$

$\mathrm{X} \quad \mathrm{X}$

$\mathrm{X}$

$\mathrm{X}$

X

X X

X X

X X

$\mathrm{X} \quad \mathrm{X}$

$\mathrm{X}$
$\mathrm{X}$
$\mathrm{X} \quad \mathrm{X}$

$\mathrm{X}$

X X

$\mathrm{X}$

X $\mathrm{X}$

X X

\footnotetext{
*The asterisk indicates species whose type locality is within Manu NP or its buffer zone.
} 
Table 1. Continued...

\begin{tabular}{|c|c|c|c|c|c|c|c|c|c|c|c|}
\hline \multirow[t]{2}{*}{ Family } & \multirow[t]{2}{*}{ Species } & \multicolumn{10}{|c|}{ Habitat type } \\
\hline & & FP & TF & LA & RI & BF & SW & SM & $\mathbf{C F}$ & MS & PU \\
\hline & Dendropsophus delarivai (Köhler and Lötters, 2001) & $\mathrm{X}$ & $\mathrm{X}$ & & & & & & & & \\
\hline & Dendropsophus koechlini (Duellman \& Trueb, 1989) & $\mathrm{X}$ & & & & & & & & & \\
\hline & Dendropsophus leali (Bokermann, 1964) & $\mathrm{X}$ & $\mathrm{X}$ & & & & & & & & \\
\hline & Dendropsophus leucophyllatus (Beireis, 1783) & $\mathrm{X}$ & $\mathrm{X}$ & & & & $\mathrm{X}$ & $\mathrm{X}$ & & & \\
\hline & Dendropsophus marmoratus (Laurenti, 1768) & $\mathrm{X}$ & $\mathrm{X}$ & & & & & & & & \\
\hline & Dendropsophus minutus (Peters, 1872) & $\mathrm{X}$ & $\mathrm{X}$ & & & $\mathrm{X}$ & $\mathrm{X}$ & $\mathrm{X}$ & & & \\
\hline & Dendropsophus parviceps (Boulenger, 1882) & $\mathrm{X}$ & $\mathrm{X}$ & & & & $\mathrm{X}$ & $\mathrm{X}$ & & & \\
\hline & Dendropsophus rhodopeplus (Günther, 1858) & $\mathrm{X}$ & $\mathrm{X}$ & & & & $\mathrm{X}$ & $\mathrm{X}$ & & & \\
\hline & Dendropsophus riveroi (Cochran \& Goin, 1970) & $\mathrm{X}$ & $\mathrm{X}$ & & & & & & & & \\
\hline & Dendropsophus rossalleni (Goin, 1959) & $\mathrm{X}$ & $\mathrm{X}$ & & & & & & & & \\
\hline & Dendropsophus sarayacuensis (Shreve, 1935) & $\mathrm{X}$ & $\mathrm{X}$ & & & & $\mathrm{X}$ & $\mathrm{X}$ & & & \\
\hline & Dendropsophus schubarti (Bokermann, 1963) & $\mathrm{X}$ & $\mathrm{X}$ & & & $\mathrm{X}$ & $\mathrm{X}$ & & & & \\
\hline & Dendropsophus triangulum (Günther, 1869) & $\mathrm{X}$ & $\mathrm{X}$ & $\mathrm{X}$ & & & & & & & \\
\hline & Hyloscirtus armatus (Boulenger, 1902) & & & & & & & $\mathrm{X}$ & $\mathrm{X}$ & & \\
\hline & Hyloscirtus phyllognathus (Melin, 1941) & & & & & & & $\mathrm{X}$ & $\mathrm{X}$ & & \\
\hline & Hypsiboas boans (Linnaeus, 1758) & $\mathrm{X}$ & $\mathrm{X}$ & & $\mathrm{X}$ & & & $\mathrm{X}$ & & & \\
\hline & Hypsiboas calcaratus (Troschel, 1848) & $\mathrm{X}$ & $\mathrm{X}$ & & & & $\mathrm{X}$ & $\mathrm{X}$ & & & \\
\hline & Hypsiboas cinerascens (Spix, 1824) & $\mathrm{X}$ & $\mathrm{X}$ & & & & $\mathrm{X}$ & $\mathrm{X}$ & & & \\
\hline & Hypsiboas fasciatus (Günther, 1858) & $\mathrm{X}$ & $\mathrm{X}$ & & & $\mathrm{X}$ & $\mathrm{X}$ & $\mathrm{X}$ & & & \\
\hline & Hypsiboas geographicus (Spix, 1824) & $\mathrm{X}$ & $\mathrm{X}$ & $\mathrm{X}$ & & $\mathrm{X}$ & $\mathrm{X}$ & $\mathrm{X}$ & & & \\
\hline & $\begin{array}{l}\text { Hypsiboas gladiator Köhler, Koscinski, Padial, Chaparro, } \\
\text { Handford, Lougheed, and De la Riva, } 2010\end{array}$ & & & & & & & $\mathrm{X}$ & $\mathrm{X}$ & & \\
\hline & Hypsiboas lanciformis Cope, 1871 "1870” & $\mathrm{X}$ & $\mathrm{X}$ & & & $\mathrm{X}$ & $\mathrm{X}$ & $\mathrm{X}$ & & & \\
\hline & Hypsiboas punctatus (Schneider, 1799) & $\mathrm{X}$ & $\mathrm{X}$ & $\mathrm{X}$ & & & & $\mathrm{X}$ & & & \\
\hline & $\begin{array}{l}\text { Osteocephalus castaneicola Moravec, Aparicio, Guerrero- } \\
\text { Reinhard, Calderón, Jungfer, and Gvoždík, } 2009\end{array}$ & $\mathrm{X}$ & $\mathrm{X}$ & & & $\mathrm{X}$ & & $X$ & & & \\
\hline & Osteocephalus buckleyi (Boulenger, 1882) & $\mathrm{X}$ & $\mathrm{X}$ & & & & $\mathrm{X}$ & & & & \\
\hline & Osteocephalus cf. leprieurii (Duméril \& Bibron, 1841) & $\mathrm{X}$ & $\mathrm{X}$ & & & $\mathrm{X}$ & & & & & \\
\hline & Osteocephalus mimeticus (Melin, 1941) & & & & & & & $\mathrm{X}$ & & & \\
\hline & Osteocephalus taurinus Steindachner, 1862 & $\mathrm{X}$ & $\mathrm{X}$ & & & $\mathrm{X}$ & $\mathrm{X}$ & & & & \\
\hline & Phyllomedusa atelopoides Duellman, Cadle \& Cannatella, 1988 & $\mathrm{X}$ & $\mathrm{X}$ & & & & & & & & \\
\hline & Phyllomedusa bicolor (Boddaert, 1772) & $\mathrm{X}$ & & & & & & & & & \\
\hline & Phyllomedusa camba De la Riva, 1999 & $\mathrm{X}$ & $\mathrm{X}$ & & & & & & & & \\
\hline & Phyllomedusa palliata Peters, 1873 & $\mathrm{X}$ & $\mathrm{X}$ & & & & $\mathrm{X}$ & & & & \\
\hline & Phyllomedusa tomopterna (Cope, 1868) & $\mathrm{X}$ & $\mathrm{X}$ & & & & & & & & \\
\hline & Phyllomedusa vaillantii Boulenger, 1882 & $\mathrm{X}$ & $\mathrm{X}$ & & & & & $\mathrm{X}$ & & & \\
\hline & Scarthyla goinorum (Bokermann, 1962) & $\mathrm{X}$ & & & & $\mathrm{X}$ & $\mathrm{X}$ & & & & \\
\hline & Scinax chiquitanus (De la Riva, 1990) & $\mathrm{X}$ & $\mathrm{X}$ & & & & & & & & \\
\hline & Scinax garbei (Miranda-Ribeiro, 1926) & $\mathrm{X}$ & $\mathrm{X}$ & & & & $\mathrm{X}$ & & & & \\
\hline & Scinax ictericus Duellman \& Wiens, 1993 & $\mathrm{X}$ & $\mathrm{X}$ & & & $\mathrm{X}$ & $\mathrm{X}$ & & & & \\
\hline & Scinax pedromedinae (Henle, 1991) & $\mathrm{X}$ & $\mathrm{X}$ & & & & & & & & \\
\hline & Scinax ruber (Laurenti, 1768) & $\mathrm{X}$ & $\mathrm{X}$ & & & $\mathrm{X}$ & $\mathrm{X}$ & $\mathrm{X}$ & & & \\
\hline & Sphaenorhynchus dorisae (Goin, 1957) & $\mathrm{X}$ & & $\mathrm{X}$ & & & & & & & \\
\hline & Sphaenorhynchus lacteus (Daudin, 1800) & $\mathrm{X}$ & & $\mathrm{X}$ & & & & & & & \\
\hline & Trachycephalus coriaceus (Peters, 1867) & $\mathrm{X}$ & $\mathrm{X}$ & & & & & & & & \\
\hline & Trachycephalus resinifictrix (Goeldi, 1907) & $\mathrm{X}$ & $\mathrm{X}$ & & & & & & & & \\
\hline & Trachycephalus venulosus (Laurenti, 1768) & $\mathrm{X}$ & $\mathrm{X}$ & & & $\mathrm{X}$ & & & & & \\
\hline LEIUPERIDAE & Edalorhina perezi Jimenez de la Espada, 1870 & $\mathrm{X}$ & $\mathrm{X}$ & & & & & & & & \\
\hline & Engystomops freibergi (Donoso-Barros, 1969) & $\mathrm{X}$ & $\mathrm{X}$ & & & $\mathrm{X}$ & & $\mathrm{X}$ & & & \\
\hline & Pleurodema marmoratum (Duméril \& Bibron, 1840) & & & & & & & & & & $\mathrm{X}$ \\
\hline LEPTODACTYLIDAE & Leptodactylus andreae Müller, 1923 & $\mathrm{X}$ & $\mathrm{X}$ & & & & & $\mathrm{X}$ & $\mathrm{X}$ & & \\
\hline & Leptodactylus bolivianus Boulenger, 1898 & $\mathrm{X}$ & $\mathrm{X}$ & & & & & & & & \\
\hline
\end{tabular}

*The asterisk indicates species whose type locality is within Manu NP or its buffer zone. 
Catenazzi, A. et al.

Table 1. Continued...

\begin{tabular}{|c|c|c|c|c|c|c|c|c|c|c|c|}
\hline \multirow[t]{2}{*}{ Family } & \multirow[t]{2}{*}{ Species } & \multicolumn{10}{|c|}{ Habitat type } \\
\hline & & FP & TF & $\mathbf{L A}$ & RI & BF & SW & SM & $\mathbf{C F}$ & MS & PU \\
\hline & $\begin{array}{l}\text { Leptodactylus didymus Heyer, Garcia-Lopez \& Cardoso, } \\
1996\end{array}$ & $\mathrm{X}$ & $\mathrm{X}$ & & & & & & & & \\
\hline & Leptodactylus griseigularis (Henle, 1981) & & & & & & & $\mathrm{X}$ & & & \\
\hline & Leptodactylus hylaedactylus (Cope, 1868) & $\mathrm{X}$ & & & & & & & & & \\
\hline & Leptodactylus knudseni Heyer, 1972 & $\mathrm{X}$ & $\mathrm{X}$ & & & & & $\mathrm{X}$ & & & \\
\hline & Leptodactylus leptodactyloides (Andersson, 1945) & $\mathrm{X}$ & $\mathrm{X}$ & & & & & & & & \\
\hline & Leptodactylus lineatus (Schneider, 1799) & $\mathrm{X}$ & $\mathrm{X}$ & & & & & & & & \\
\hline & Leptodactylus pentadactylus (Laurenti, 1768) & $\mathrm{X}$ & $\mathrm{X}$ & & & $\mathrm{X}$ & & $\mathrm{X}$ & & & \\
\hline & Leptodactylus petersii (Steindachner, 1864) & $\mathrm{X}$ & $\mathrm{X}$ & & & $\mathrm{X}$ & $\mathrm{X}$ & $\mathrm{X}$ & & & \\
\hline & Leptodactylus rhodomystax Boulenger, 1884 “1883” & $\mathrm{X}$ & $\mathrm{X}$ & & & & $\mathrm{X}$ & $\mathrm{X}$ & & & \\
\hline & Leptodactylus rhodonotus (Günther, 1868) & $\mathrm{X}$ & $\mathrm{X}$ & & & & & $\mathrm{X}$ & & & \\
\hline & Leptodactylus stenodema Jiménez de la Espada, 1875 & $\mathrm{X}$ & $\mathrm{X}$ & & & & & & & & \\
\hline \multirow{7}{*}{ MICROHYLIDAE } & Altigius alios Wild, 1995 & $\mathrm{X}$ & & & & & & & & & \\
\hline & Chiasmocleis bassleri Dunn, 1949 & $\mathrm{X}$ & $\mathrm{X}$ & & & & & & & & \\
\hline & Chiasmocleis ventrimaculata (Andersson, 1945) & $\mathrm{X}$ & $\mathrm{X}$ & & & $\mathrm{X}$ & $\mathrm{X}$ & & & & \\
\hline & Ctenophryne geayi Mocquard, 1904 & $\mathrm{X}$ & $\mathrm{X}$ & & & & & & & & \\
\hline & Elachistocleis muiraquitan Nunes de Almeida \& Toledo, 2012 & $\mathrm{X}$ & & & & & & & & & \\
\hline & Hamptophryne boliviana (Parker, 1927) & $\mathrm{X}$ & $\mathrm{X}$ & & & & & & & & \\
\hline & Syncope antenori Walker, 1973 & $\mathrm{X}$ & $\mathrm{X}$ & & & $\mathrm{X}$ & & & & & \\
\hline PIPIDAE & Pipa pipa (Linnaeus, 1758) & $\mathrm{X}$ & & & & & $\mathrm{X}$ & & & & \\
\hline \multirow[t]{30}{*}{ STRABOMANTIDAE } & *Bryophryne cophites (Lynch, 1975) & & & & & & & & & $\mathrm{X}$ & $\mathrm{X}$ \\
\hline & *Bryophryne nubilosus Lehr \& Catenazzi, 2008 & & & & & & & & $\mathrm{X}$ & $\mathrm{X}$ & \\
\hline & *Bryophryne hanssaueri Lehr \& Catenazzi, 2009 & & & & & & & & $\mathrm{X}$ & & \\
\hline & Noblella sp. & & & & & & & & $\mathrm{X}$ & & \\
\hline & Noblella cf. myrmecoides (Lynch, 1976) & $\mathrm{X}$ & $\mathrm{X}$ & & & $\mathrm{X}$ & $\mathrm{X}$ & & & & \\
\hline & *Noblella pygmaea Lehr \& Catenazzi, 2009 & & & & & & & & $\mathrm{X}$ & $\mathrm{X}$ & $\mathrm{X}$ \\
\hline & Oreobates cruralis (Boulenger, 1902) & $\mathrm{X}$ & $\mathrm{X}$ & & & $\mathrm{X}$ & $\mathrm{X}$ & & & & \\
\hline & $\begin{array}{l}\text { *Oreobates gemcare Padial, Chaparro, Castroviejo-Fisher, } \\
\text { Guayasamin, Lehr, Delgado, Vaira, Teixeira, Aguayo-Vedia, } \\
\text { and De la Riva, } 2012\end{array}$ & & & & & & & $\mathrm{X}$ & $\mathrm{X}$ & & \\
\hline & Oreobates granulosus (Boulenger, 1903) & & & & & & & $\mathrm{X}$ & $\mathrm{X}$ & & \\
\hline & Oreobates quixensis Jiménez de la Espada, 1872 & $\mathrm{X}$ & $\mathrm{X}$ & & & $\mathrm{X}$ & & $\mathrm{X}$ & & & \\
\hline & Pristimantis altamazonicus (Barbour \& Dunn, 1921) & $\mathrm{X}$ & $\mathrm{X}$ & & & $\mathrm{X}$ & $\mathrm{X}$ & $\mathrm{X}$ & & & \\
\hline & *Pristimantis buccinator (Rodríguez, 1994) & $\mathrm{X}$ & $\mathrm{X}$ & & & & $\mathrm{X}$ & $\mathrm{X}$ & & & \\
\hline & Pristimantis carvalhoi (Lutz in Lutz \& Kloss, 1952) & $\mathrm{X}$ & $\mathrm{X}$ & & & $\mathrm{X}$ & & & & & \\
\hline & *Pristimantis cosnipatae (Duellman, 1978) & & & & & & & $\mathrm{X}$ & $\mathrm{X}$ & & \\
\hline & Pristimantis croceoinguinis (Lynch, 1968) & $\mathrm{X}$ & $\mathrm{X}$ & & & & & & & & \\
\hline & *Pristimantis danae (Duellman, 1978) & & & & & & & $\mathrm{X}$ & $\mathrm{X}$ & $\mathrm{X}$ & \\
\hline & Pristimantis diadematus (Jimenez de la Espada, 1875) & $\mathrm{X}$ & $\mathrm{X}$ & & & & & & & & \\
\hline & Pristimantis divnae Lehr \& von May, 2009 & & $\mathrm{X}$ & & & $\mathrm{X}$ & & & & & \\
\hline & Pristimantis fenestratus (Steindachner, 1864) & $\mathrm{X}$ & & & $\mathrm{X}$ & & & & & & \\
\hline & Pristimantis imitatrix (Duellman, 1978) & $\mathrm{X}$ & $\mathrm{X}$ & & & & & & & & \\
\hline & Pristimantis lacrimosus (Jimenez de la Espada, 1875) & $\mathrm{X}$ & $\mathrm{X}$ & & & & & & & & \\
\hline & *Pristimantis lindae (Duellman, 1978) & & & & & & & $\mathrm{X}$ & $\mathrm{X}$ & & \\
\hline & Pristimantis mendax (Duellman, 1978) & $\mathrm{X}$ & $\mathrm{X}$ & & & & & & & & \\
\hline & Pristimantis mercedesae (Lynch \& McDiarmid, 1987) & & & & & & & $\mathrm{X}$ & $\mathrm{X}$ & & \\
\hline & Pristimantis ockendeni (Boulenger, 1912) & $\mathrm{X}$ & $\mathrm{X}$ & & & $\mathrm{X}$ & & & & & \\
\hline & $\begin{array}{l}\text { Pristimantis olivaceus (Köhler, Morales, Lötters, Reichle } \\
\text { \& Aparicio, 1998) }\end{array}$ & $\mathrm{X}$ & $\mathrm{X}$ & & & & & $\mathrm{X}$ & & & \\
\hline & *Pristimantis pharangobates (Duellman, 1978) & & & & & & & $\mathrm{X}$ & $\mathrm{X}$ & $\mathrm{X}$ & \\
\hline & Pristimantis platydactylus (Boulenger, 1903) & & & & & & & & $\mathrm{X}$ & & \\
\hline & Pristimantis reichlei Padial \& De la Riva, 2009 & $\mathrm{X}$ & $\mathrm{X}$ & & & $\mathrm{X}$ & $\mathrm{X}$ & $\mathrm{X}$ & $\mathrm{X}$ & & \\
\hline & *Pristimantis salaputium (Duellman, 1978) & & & & & & & $\mathrm{X}$ & $\mathrm{X}$ & & \\
\hline
\end{tabular}

\footnotetext{
*The asterisk indicates species whose type locality is within Manu NP or its buffer zone.
} 
The herpetofauna of Manu National Park, Peru

Table 1. Continued...

\begin{tabular}{|c|c|c|c|c|c|c|c|c|c|c|c|}
\hline \multirow[t]{2}{*}{ Family } & \multirow[t]{2}{*}{ Species } & \multicolumn{10}{|c|}{ Habitat type } \\
\hline & & FP & TF & LA & RI & BF & SW & SM & $\mathbf{C F}$ & MS & PU \\
\hline & *Pristimantis skydmainos (Flores \& Rodriguez, 1997) & $\mathrm{X}$ & & & & & & & & & \\
\hline & $\begin{array}{l}\text { Pristimantis tantanti (Lehr, Torres-Gastello \& Suárez- } \\
\text { Segovia, 2007) }\end{array}$ & & $\mathrm{X}$ & & & & & & & & \\
\hline & Pristimantis toftae (Duellman, 1978) & $\mathrm{X}$ & $\mathrm{X}$ & & & $\mathrm{X}$ & $\mathrm{X}$ & $\mathrm{X}$ & $\mathrm{X}$ & & \\
\hline & Pristimantis ventrimarmoratus (Boulenger, 1912) & $\mathrm{X}$ & $\mathrm{X}$ & & & & & & & & \\
\hline & Psychrophrynella sp. 1 & & & & & & & & $\mathrm{X}$ & $\mathrm{X}$ & \\
\hline & Psychrophrynella sp. 2 & & & & & & & & $\mathrm{X}$ & & \\
\hline & $\begin{array}{l}\text { *Psychrophrynella usurpator De la Riva, Chaparro \& } \\
\text { Padial, } 2008\end{array}$ & & & & & & & & $\mathrm{X}$ & $\mathrm{X}$ & $\mathrm{X}$ \\
\hline & Strabomantis sulcatus (Cope, 1874) & $\mathrm{X}$ & $\mathrm{X}$ & & & $\mathrm{X}$ & & $\mathrm{X}$ & & & \\
\hline Order Caudata & & & & & & & & & & & \\
\hline $\begin{array}{l}\text { PLETHODONTIDAE } \\
\text { Order Gymnophiona }\end{array}$ & Bolitoglossa altamazonica (Cope, 1874) & $\mathrm{X}$ & & & & $\mathrm{X}$ & $\mathrm{X}$ & $\mathrm{X}$ & & & \\
\hline CAECILIIDAE & Oscaecilia bassleri (Dunn, 1942) & $\mathrm{X}$ & & & & & & & & & \\
\hline & Siphonops annulatus (Mikan, 1820) & & $\mathrm{X}$ & & & & & & & & \\
\hline RHINATREMATIDAE & Epicrionops bicolor Boulenger, 1883 & & & & & & & $\mathrm{X}$ & $\mathrm{X}$ & & \\
\hline
\end{tabular}

*The asterisk indicates species whose type locality is within Manu NP or its buffer zone.

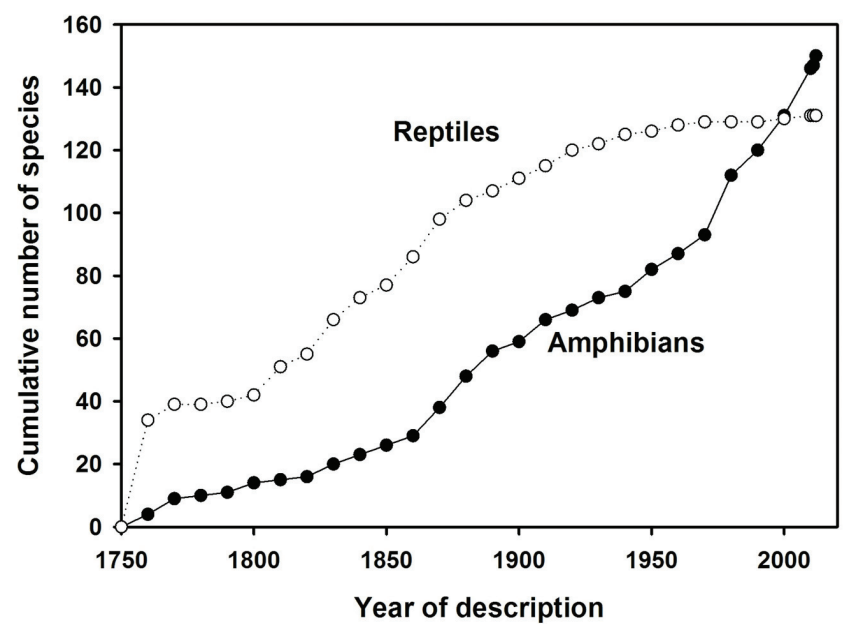

Figure 2. Cumulative number of amphibian and reptile species known from Manu National Park and its buffer zone as a function of year of description.

turtle species. Although covering approximately $0.01 \%$ of the planet's terrestrial surface, this protected area preserves $2.2 \%$ of the amphibian and $1.5 \%$ of the reptilian diversity (considering extant taxa only).

However, the montane amphibian fauna within the park and the adjacent Kosñipata valley has recently experienced dramatic declines and local extinctions (Catenazzi et al. 2011). The collapse of amphibian species richness and abundance has been more pronounced at mid-elevations (1200-2000 m) and for stream-breeding species (Catenazzi et al. 2011). These declines occurred over less than a decade and coincided with the arrival of the pathogenic fungus Batrachochytrium dendrobatidis $(\mathrm{Bd})$ to southern Peru (Seimon et al. 2007). The sudden disappearance of a sizable proportion of the montane anuran fauna despite the excellent state of conservation of the forest and protection granted by Manu NP shows that additional conservation actions are needed to preserve amphibian biodiversity. These actions should focus on managing the impact of outbreaks of B. dendrobatidis on amphibian communities.
Material examined (all from PERU). AMPHIBIANS. Aromobatidae. Allobates alessandroi: Cusco: Provincia Paucartambo, Kosñipata, MUSM 17734-36, KU 139134. Allobates conspicuus: Madre de Dios: Provincia Manu, Los Amigos Conservation Concession, MUSM 24123, 24190, 24192, 24203, 24212, 24238-39, 24241, 24243, 24254, 24264. Allobates femoralis: Madre de Dios: Provincia Manu, Los Amigos Conservation Concession, MUSM 24233. Allobates trilineatus: Cusco: Provincia Paucartambo, Kosñipata, MHNG 2607.19-20, MUSM 17735, 21083-85; Madre de Dios: Provincia Manu, Los Amigos Conservation Concession, MUSM 24197, 24210, 24223. Bufonidae. Amazophrynella minuta: Madre de Dios: Provincia Manu, Los Amigos Conservation Concession, MUSM 24334, 27286, 27287. Rhinella inca: Cusco: Provincia Paucartambo, Kosñipata, MUSM 27878, KU 139050-52, 139431. Rhinella manu: Cusco: Provincia Paucartambo, Kosñipata, MUSM 26282, 27929-33. Rhinella margaritifera, Cusco: Provincia Paucartambo, Kosñipata, MHNG 2607.07-10, MUSM 21180-81; Madre de Dios: Provincia Manu, Los Amigos Conservation Concession, MUSM 24130, 24163-64, 24180, 24255, $24259,24297$. Rhinella veraguensis: Cusco: Provincia Paucartambo, Kosñipata, MHNG 2606.61-63, MUSM 21171, 21178-79, 30438. Caecilidae. Oscaecilia bassleri: Madre de Dios: Pakitza, MVZ 196910. Siphonops annulatus: Madre de Dios: Provincia Manu, Los Amigos Conservation Concession, MUSM 24342. Centrolenidae. Centrolene sabini: Cusco: Provincia Paucartambo, Kosñipata, MUSM 17966, 27941, 28017-28019, 30346. Hyalinobatrachium bergeri: Cusco: Provincia Paucartambo, Kosñipata, CORBIDI -HE 11917, MHNG 2606.57-60, MUSM 21130-36, 21138, 27875-77, 28032, 30436-37, KU 162248-49. Nymphargus pluvialis: Cusco: Provincia Paucartambo, Kosñipata, MHNG 2606.54-55, MUSM 17963-17965, 17967-17968, 27862, 27940. Nymphargus truebae: Cusco: Provincia Paucartambo, Kosñipata, MHNG 2606.68-70, MUSM 27970-27971, KU 16226882. Rulyrana spiculata: Cusco: Provincia Paucartambo, Kosñipata, MHNG 2602.93-95, MUSM 17973-17977, MUSM 17976-17977, KU 162283-84. Teratohyla midas: Madre de Dios: Provincia Manu, Los Amigos Conservation Concession, MUSM 24061-68. Ceratophryidae. Ceratophrys cornuta: Madre de Dios: Provincia Manu, Los Amigos Conservation Concession, MUSM 24173, 24253. 
Table 2. Reptiles of Manu National Park, Peru. See Table 1 for details.

\begin{tabular}{|c|c|c|c|c|c|c|c|c|c|c|c|}
\hline Family & Species & FP & TF & $\mathbf{L A}$ & $\mathbf{R I}$ & BF & SW & $\mathbf{S M}$ & $\mathbf{C F}$ & MS & $\mathbf{P U}$ \\
\hline \multicolumn{12}{|l|}{ Order Crocodilia } \\
\hline \multirow[t]{4}{*}{ ALLIGATORIDAE } & Caiman crocodilus (Linnaeus, 1758) & & & $\mathrm{X}$ & $\mathrm{X}$ & & $\mathrm{X}$ & & & & \\
\hline & Melanosuchus niger (Spix, 1825) & & & $\mathrm{X}$ & & & $\mathrm{X}$ & & & & \\
\hline & Paleosuchus palpebrosus (Cuvier, 1807) & $\mathrm{X}$ & $\mathrm{X}$ & $\mathrm{X}$ & & & $\mathrm{X}$ & & & & \\
\hline & Paleosuchus trigonatus (Schneider, 1801) & $\mathrm{X}$ & $\mathrm{X}$ & $\mathrm{X}$ & & & $\mathrm{X}$ & $\mathrm{X}$ & & & \\
\hline \multicolumn{12}{|l|}{ Order Squamata: Sauria } \\
\hline AMPHISBAENIDAE & Amphisbaena fuliginosa Linnaeus, 1758 & $\mathrm{X}$ & & & & & & & & & \\
\hline ANGUIDAE & Diploglossus fasciatus (Gray, 1831) & $\mathrm{X}$ & $\mathrm{X}$ & & & & & & & & \\
\hline \multirow[t]{4}{*}{ GEKKONIDAE } & $\begin{array}{l}\text { Thecadactylus solimoensis Bergman \& Russell, } \\
2007\end{array}$ & $\mathrm{X}$ & $\mathrm{X}$ & & & & & & & & \\
\hline & Gonatodes hasemani Griffin, 1917 & $\mathrm{X}$ & $\mathrm{X}$ & & & & & & & & \\
\hline & Gonatodes humeralis (Guichenot, 1855) & $\mathrm{X}$ & $\mathrm{X}$ & & & & $\mathrm{X}$ & $\mathrm{X}$ & & & \\
\hline & Pseudogonatodes guianensis Parker, 1935 & $\mathrm{X}$ & $\mathrm{X}$ & & & $\mathrm{X}$ & & & & & \\
\hline \multirow[t]{14}{*}{ GYMNOPHTHALMIDAE } & Alopoglossus angulatus (Linnaeus, 1758) & $\mathrm{X}$ & $\mathrm{X}$ & & & $\mathrm{X}$ & & & & & \\
\hline & Alopoglossus buckleyi (O’Shaughnessy, 1881) & $\mathrm{X}$ & & & & & & & & & \\
\hline & Ptychoglossus brevifrontalis Boulenger, 1912 & $\mathrm{X}$ & & & & & & & & & \\
\hline & Bachia dorbignyi Duméril \& Bibron, 1839 & $\mathrm{X}$ & $\mathrm{X}$ & & & & & & & & \\
\hline & Bachia trisanale (Cope, 1868) & $\mathrm{X}$ & $\mathrm{X}$ & & & & & & & & \\
\hline & Cercosaura argulus Peters, 1863 & $\mathrm{X}$ & $\mathrm{X}$ & & & & & & & & \\
\hline & Cercosaura eigenmanni Griffin, 1917 & $\mathrm{X}$ & $\mathrm{X}$ & & & & & & & & \\
\hline & Cercosaura manicatus O’Shaughnessy, 1881 & $X$ & & & & & & & & & \\
\hline & Cercosaura ocellata Wagler, 1830 & $\mathrm{X}$ & $\mathrm{X}$ & & & $\mathrm{X}$ & $\mathrm{X}$ & $\mathrm{X}$ & & & \\
\hline & Potamites sp. & & & & & & & $\mathrm{X}$ & $\mathrm{X}$ & & \\
\hline & Potamites ecpleopus Cope, 1875 & $\mathrm{X}$ & $\mathrm{X}$ & & & $\mathrm{X}$ & & $\mathrm{X}$ & & & \\
\hline & Proctoporus bolivianus Werner, 1910 & & & & & & & & & $\mathrm{X}$ & $\mathrm{X}$ \\
\hline & Proctoporus pachyurus Tschudi, 1845 & & & & & & & & $\mathrm{X}$ & $\mathrm{X}$ & $\mathrm{X}$ \\
\hline & Iphisa elegans Gray, 1851 & $\mathrm{X}$ & $\mathrm{X}$ & & & & & & & & \\
\hline \multirow[t]{2}{*}{ HOPLOCERCIDAE } & Enyalioides laticeps (Guichenot, 1855) & $\mathrm{X}$ & $\mathrm{X}$ & & & & & & & & \\
\hline & Enyalioides palpebralis (Boulenger, 1883) & $\mathrm{X}$ & $\mathrm{X}$ & & & & & $\mathrm{X}$ & & & \\
\hline \multirow[t]{7}{*}{ POLYCHROTIDAE } & Anolis chrysolepis Duméril \& Bibron, 1837 & $\mathrm{X}$ & & & & & & & & & \\
\hline & $\begin{array}{l}\text { *Anolis cuscoensis Poe, Yañez-Miranda \& Lehr, } \\
2008\end{array}$ & & & & & & & $\mathrm{X}$ & $\mathrm{X}$ & & \\
\hline & Anolis fuscoauratus D’Orbigny, 1837 & $\mathrm{X}$ & $\mathrm{X}$ & & & $\mathrm{X}$ & $\mathrm{X}$ & $\mathrm{X}$ & & & \\
\hline & Anolis nitens (Wagler, 1830) & $\mathrm{X}$ & $\mathrm{X}$ & & & & & & & & \\
\hline & Anolis ortonii Cope, 1869 & $\mathrm{X}$ & $\mathrm{X}$ & & & & & & & & \\
\hline & Anolis punctatus Daudin, 1802 & $\mathrm{X}$ & $\mathrm{X}$ & & & & & & & & \\
\hline & Polychrus liogaster Boulenger, 1908 & & $\mathrm{X}$ & & & & & & & & \\
\hline \multirow[t]{2}{*}{ SCINCIDAE } & Copeoglossum nigropunctatum Spix, 1825 & $\mathrm{X}$ & $\mathrm{X}$ & & & & $\mathrm{X}$ & & & & \\
\hline & Exila nigropalmata Andersson, 1918 & & $\mathrm{X}$ & & & & & & & & \\
\hline \multirow[t]{5}{*}{ TEIIDAE } & Ameiva ameiva (Linnaeus, 1758) & $\mathrm{X}$ & $\mathrm{X}$ & & & & & & & & \\
\hline & Dracaena guianensis Daudin, 1802 & $X$ & $\mathrm{X}$ & & & & & & & & \\
\hline & Kentropyx altamazonica Cope, 1876 & & $\mathrm{X}$ & & & & & & & & \\
\hline & Kentropyx pelviceps Cope, 1868 & $\mathrm{X}$ & $\mathrm{X}$ & & & $\mathrm{X}$ & $\mathrm{X}$ & $\mathrm{X}$ & & & \\
\hline & Tupinambis teguixin Linnaeus, 1758 & $\mathrm{X}$ & $\mathrm{X}$ & & & & $\mathrm{X}$ & & & & \\
\hline \multirow[t]{6}{*}{ TROPIDURIDAE } & Stenocercus fimbriatus Avila-Pires, 1995 & $\mathrm{X}$ & $\mathrm{X}$ & & & & & & & & \\
\hline & Stenocerus roseiventris Duméril \& Bibron ,1837 & $\mathrm{X}$ & $\mathrm{X}$ & & & $\mathrm{X}$ & & & & & \\
\hline & Plica plica (Linnaeus, 1758) & $\mathrm{X}$ & $\mathrm{X}$ & & & $\mathrm{X}$ & & & & & \\
\hline & Plica umbra (Linnaeus, 1758) & $\mathrm{X}$ & $\mathrm{X}$ & & & & $\mathrm{X}$ & & & & \\
\hline & Uracentron azureum Linnaeus, 1758 & & $\mathrm{X}$ & & & & $\mathrm{X}$ & & & & \\
\hline & Uracentron flaviceps (Guichenot, 1855) & $\mathrm{X}$ & $\mathrm{X}$ & & & & & & & & \\
\hline \multicolumn{12}{|l|}{ Order Squamata: Serpentes } \\
\hline ANILIIDAE & Anilius scytale (Linnaeus, 1758) & $\mathrm{X}$ & $\mathrm{X}$ & & & & & $\mathrm{X}$ & & & \\
\hline \multirow[t]{2}{*}{ BOIDAE } & Boa constrictor Linnaeus, 1758 & $\mathrm{X}$ & $\mathrm{X}$ & & & & $\mathrm{X}$ & & & & \\
\hline & Corallus batesi (Gray, 1860) & $\mathrm{X}$ & $\mathrm{X}$ & & & & & & & & \\
\hline
\end{tabular}

\footnotetext{
*The asterisk indicates species whose type locality is within Manu NP or its buffer zone.
} 
Table 2. Continued...

\begin{tabular}{|c|c|c|c|c|c|c|c|c|c|c|c|}
\hline Family & Species & FP & TF & $\mathbf{L A}$ & RI & BF & SW & $\mathbf{S M}$ & $\mathbf{C F}$ & MS & $\mathbf{P U}$ \\
\hline & Corallus hortulanus (Linnaeus, 1758) & $\mathrm{X}$ & $\mathrm{X}$ & & & & $\mathrm{X}$ & & & & \\
\hline & Epicrates cenchria (Linnaeus, 1758) & $\mathrm{X}$ & $\mathrm{X}$ & & & & & & & & \\
\hline & Eunectes murinus Linnaeus, 1758 & $\mathrm{X}$ & $\mathrm{X}$ & & & & $\mathrm{X}$ & & & & \\
\hline \multirow[t]{51}{*}{ COLUBRIDAE } & Apostolepis nigroterminata Boulenger, 1896 & & & & & & & $\mathrm{X}$ & & & \\
\hline & Atractus elaps (Günther, 1858) & & $\mathrm{X}$ & & & & & & & & \\
\hline & Atractus flammigerus Boie, 1827 & & $\mathrm{X}$ & & & & & & & & \\
\hline & Atractus major Boulenger, 1894 & & $\mathrm{X}$ & & & & & & & & \\
\hline & Chironius carinatus (Linnaeus, 1758) & & & & & & & $\mathrm{X}$ & & & \\
\hline & Chironius exoletus (Linnaeus, 1758) & $\mathrm{X}$ & $\mathrm{X}$ & & & & & & & & \\
\hline & Chironius fuscus (Linnaeus, 1758) & $\mathrm{X}$ & $\mathrm{X}$ & & & & & $\mathrm{X}$ & & & \\
\hline & Chironius monticola Roze, 1952 & & & & & & & $\mathrm{X}$ & $\mathrm{X}$ & $\mathrm{X}$ & \\
\hline & Chironius cf. multiventris Schmidt \& Walker, 1942 & $\mathrm{X}$ & & & & & & & & & \\
\hline & Chironius scurrulus Wagler, 1824 & $\mathrm{X}$ & $\mathrm{X}$ & & & & & & & & \\
\hline & Clelia clelia (Daudin, 1803) & $\mathrm{X}$ & $\mathrm{X}$ & & & & & $\mathrm{X}$ & & & \\
\hline & Dendrophidion dendrophis Schlegel, 1837 & $\mathrm{X}$ & $\mathrm{X}$ & & & & & & & & \\
\hline & Dipsas catesbyi (Sentzen, 1796) & $\mathrm{X}$ & $\mathrm{X}$ & & & & $\mathrm{X}$ & $\mathrm{X}$ & & & \\
\hline & Dipsas indica Laurenti, 1768 & $\mathrm{X}$ & & & & & $\mathrm{X}$ & & & & \\
\hline & Dipsas peruana (Boettger, 1898) & & & & & & & $\mathrm{X}$ & $\mathrm{X}$ & & \\
\hline & Dipsas variegata Duméril, Bibron \& Duméril, 1854 & & & & & & & & $\mathrm{X}$ & & \\
\hline & Drepanoides anomalus (Jan, 1863) & $\mathrm{X}$ & $\mathrm{X}$ & & & & $\mathrm{X}$ & & & & \\
\hline & Drymarchon corais Boie, 1827 & $\mathrm{X}$ & $\mathrm{X}$ & & & & & & & & \\
\hline & Drymobius rhombifer Günther, 1860 & $\mathrm{X}$ & & & & & & & & & \\
\hline & Drymoluber dichrous (Peters, 1863) & $\mathrm{X}$ & $\mathrm{X}$ & & & & & & & & \\
\hline & Helicops angulatus (Linnaeus, 1758) & $\mathrm{X}$ & $\mathrm{X}$ & $\mathrm{X}$ & & & $\mathrm{X}$ & & & & \\
\hline & Helicops leopardinus Schlegel, 1837 & $\mathrm{X}$ & $\mathrm{X}$ & & & & $\mathrm{X}$ & & & & \\
\hline & Helicops polylepis Günther, 1861 & $\mathrm{X}$ & $\mathrm{X}$ & & & & & & & & \\
\hline & Imantodes cenchoa (Linnaeus, 1758) & $\mathrm{X}$ & $\mathrm{X}$ & & & $\mathrm{X}$ & $\mathrm{X}$ & $\mathrm{X}$ & & & \\
\hline & Imantodes lentiferus Cope, 1894 & $\mathrm{X}$ & $\mathrm{X}$ & & & & $\mathrm{X}$ & & & & \\
\hline & Leptodeira annulata (Linnaeus, 1758) & $\mathrm{X}$ & $\mathrm{X}$ & & & $\mathrm{X}$ & $\mathrm{X}$ & $\mathrm{X}$ & & & \\
\hline & Leptophis ahaetulla (Linnaeus, 1758) & $\mathrm{X}$ & $\mathrm{X}$ & & & & & & & & \\
\hline & Liophis reginae (Linnaeus, 1758) & $\mathrm{X}$ & $\mathrm{X}$ & & & & & & & & \\
\hline & Liophis taeniurus Tschudi, 1845 & & & & & & & $\mathrm{X}$ & $\mathrm{X}$ & & \\
\hline & Liophis taeniogaster Jan, 1866 & $\mathrm{X}$ & $\mathrm{X}$ & & & & $\mathrm{X}$ & $\mathrm{X}$ & & & \\
\hline & Liophis typhlus (Linnaeus, 1758) & & $\mathrm{X}$ & & & & & & & & \\
\hline & Ninia hudsoni Parker, 1940 & $\mathrm{X}$ & $\mathrm{X}$ & & & & & & & & \\
\hline & Oxybelis aeneus (Wagler, 1824) & $\mathrm{X}$ & $\mathrm{X}$ & & & & & & & & \\
\hline & Oxybelis fulgidus (Daudin, 1803) & $\mathrm{X}$ & $\mathrm{X}$ & & & & & & & & \\
\hline & Oxyrhopus erdisii Barbour, 1913 & & $\mathrm{X}$ & & & & & & & & \\
\hline & Oxyrhopus formosus Wied, 1820 & $\mathrm{X}$ & $\mathrm{X}$ & & & & & & & & \\
\hline & Oxyrhopus marcapatae Boulenger 1902 & & & & & & & $\mathrm{X}$ & $\mathrm{X}$ & & \\
\hline & Oxyrhopus melanogenys (Tschudi, 1845) & $\mathrm{X}$ & $\mathrm{X}$ & & & $\mathrm{X}$ & & & & & \\
\hline & Oxyrhopus petola (Linnaeus, 1758) & $\mathrm{X}$ & $\mathrm{X}$ & & & & & $\mathrm{X}$ & & & \\
\hline & Pseustes poecilonotus Günther, 1858 & & & & & & & & & & \\
\hline & Pseustes sulphureus Wagler, 1824 & $\mathrm{X}$ & $\mathrm{X}$ & & & & & & & & \\
\hline & Philodryas viridissimus (Linnaeus, 1758) & & $\mathrm{X}$ & & & & & & & & \\
\hline & Pseudoboa coronata Schneider, 1801 & & $\mathrm{X}$ & & & & & & & & \\
\hline & Pseudoeryx plicatilis (Linnaeus, 1758) & $\mathrm{X}$ & & & & & $\mathrm{X}$ & & & & \\
\hline & Rhinobothryum lentiginosum Scopoli, 1785 & $\mathrm{X}$ & $\mathrm{X}$ & & & & & & & & \\
\hline & Siphlophis cervinus Laurenti, 1768 & $\mathrm{X}$ & $\mathrm{X}$ & & & $\mathrm{X}$ & & & & & \\
\hline & Siphlophis compressus Daudin, 1803 & $\mathrm{X}$ & $\mathrm{X}$ & & & $\mathrm{X}$ & & & & & \\
\hline & Spilotes pullatus (Linnaeus, 1758) & $\mathrm{X}$ & $\mathrm{X}$ & & & & & & & & \\
\hline & Tachymenis peruviana Wiegmann, 1835 & & & & & & & & & $\mathrm{X}$ & $\mathrm{X}$ \\
\hline & Taeniophallus brevirostris Peters, 1863 & $\mathrm{X}$ & $\mathrm{X}$ & & & & & & & & \\
\hline & Taeniophallus occipitalis Jan, 1863 & & $\mathrm{X}$ & & & & & & & & \\
\hline
\end{tabular}

*The asterisk indicates species whose type locality is within Manu NP or its buffer zone. 
Table 2. Continued...

\begin{tabular}{|c|c|c|c|c|c|c|c|c|c|c|c|}
\hline Family & Species & FP & TF & $\mathbf{L A}$ & RI & $\mathbf{B F}$ & SW & SM & $\mathbf{C F}$ & MS & $\mathbf{P U}$ \\
\hline & Tantilla melanocephala (Linnaeus, 1758) & & $\mathrm{X}$ & & & & & & & & \\
\hline & Xenodon rabdocephalus (Wied, 1824) & $\mathrm{X}$ & $\mathrm{X}$ & & & & & & & & \\
\hline & Xenodon severus (Linnaeus, 1758) & $\mathrm{X}$ & $\mathrm{X}$ & & & & & & & & \\
\hline & Xenoxybelis argenteus Daudin, 1803 & $\mathrm{X}$ & & & & & & & & & \\
\hline & Xenoxybelis boulengeri Procter, 1923 & & $\mathrm{X}$ & & & & & & & & \\
\hline & Xenopholis scalaris Wucherer, 1861 & $\mathrm{X}$ & $\mathrm{X}$ & & & & & & & & \\
\hline \multirow[t]{5}{*}{ ELAPIDAE } & Micrurus annellatus (Peters, 1871) & & & & & & & $\mathrm{X}$ & $\mathrm{X}$ & & \\
\hline & Micrurus lemniscatus (Linnaeus, 1758) & $\mathrm{X}$ & $\mathrm{X}$ & & & & $\mathrm{X}$ & $\mathrm{X}$ & & & \\
\hline & Micrurus narduccii (Jan, 1863) & & $\mathrm{X}$ & & & & & $\mathrm{X}$ & & & \\
\hline & Micrurus obscurus Jan, 1872 & $\mathrm{X}$ & $\mathrm{X}$ & & & & & & & & \\
\hline & Micrurus surinamensis Cuvier, 1817 & $\mathrm{X}$ & $\mathrm{X}$ & & & & & $\mathrm{X}$ & & & \\
\hline LEPTOTYPHLOPIDAE & Leptotyphlops cf. diaplocius Oreja-Miranda, 1969 & & $\mathrm{X}$ & & & & & & & & \\
\hline TYPHLOPIDAE & Typhlops reticulatus Linnaeus, 1758 & $\mathrm{X}$ & $\mathrm{X}$ & & & & & & & & \\
\hline \multirow[t]{8}{*}{ VIPERIDAE } & Bothriopsis bilineata (Wied, 1825) & $\mathrm{X}$ & $\mathrm{X}$ & & & $\mathrm{X}$ & & $\mathrm{X}$ & & & \\
\hline & Bothriopsis oligolepis (Werner 1901) & & & & & & & & $\mathrm{X}$ & & \\
\hline & Bothriopsis taeniata Wagler, 1824 & & $\mathrm{X}$ & & & & & $\mathrm{X}$ & & & \\
\hline & Bothrocophias microphthalmus (Cope, 1876) & & $\mathrm{X}$ & & & & & & & & \\
\hline & Bothrops andianus Amaral, 1923 & & & & & & & $\mathrm{X}$ & $\mathrm{X}$ & & \\
\hline & Bothrops atrox (Linnaeus, 1758) & $\mathrm{X}$ & $\mathrm{X}$ & & $\mathrm{X}$ & & $\mathrm{X}$ & $\mathrm{X}$ & & & \\
\hline & Bothrops brazili Hoge, 1954 & $\mathrm{X}$ & $\mathrm{X}$ & & & & & & & & \\
\hline & Lachesis muta (Linnaeus, 1766) & $\mathrm{X}$ & $\mathrm{X}$ & & & & $\mathrm{X}$ & $\mathrm{X}$ & & & \\
\hline \multicolumn{12}{|l|}{ Order Testudinata } \\
\hline KINOSTERNIDAE & Kinosternon scorpioides Linnaeus, 1766 & $\mathrm{X}$ & & & & & & & & & \\
\hline \multirow[t]{5}{*}{ CHELIDAE } & Chelus fimbriatus (Schneider, 1873) & $\mathrm{X}$ & & & & & & & & & \\
\hline & Mesoclemmys gibba (Schweigger, 1812) & & & & $\mathrm{X}$ & & & & & & \\
\hline & Mesoclemmys raniceps (Gray, 1855) & & & & $\mathrm{X}$ & & & & & & \\
\hline & Phrynops geoffranus (Schweigger, 1812) & & & & $\mathrm{X}$ & & & & & & \\
\hline & Platemys platycephala (Schneider, 1792) & $\mathrm{X}$ & & & & & & & & & \\
\hline PODOCNEMIDAE & Podocnemis unifilis Troschel, 1848 & & & $\mathrm{X}$ & $\mathrm{X}$ & & & & & & \\
\hline TESTUDINAE & Chelonoidis denticulata (Linnaeus, 1766) & $\mathrm{X}$ & $\mathrm{X}$ & & & & & & & & \\
\hline
\end{tabular}

*The asterisk indicates species whose type locality is within Manu NP or its buffer zone.

Telmatobius mendelsoni: Cusco: Provincia Paucartambo, Kosñipata, MHNG 2606.48-49, MUSM 17969, 21149, $21150,26285$. Telmatobius timens: Cusco: Provincia Paucartambo, Kosñipata, KU 139040-41. Dendrobatidae. Ameerega hahneli: Madre de Dios: Provincia Manu, Los Amigos Conservation Concession, MUSM 24179, 24182, 24187, 24201, 24235, 24312, 24316. Ameerega macero: Cusco: Provincia Paucartambo, Kosñipata, MHNG 2607.44. Ameerega simulans: MHNG 2607.04-06, MUSM 21006-7, 21111-15. Ameerega trivittata: Madre de Dios: Provincia Manu, Los Amigos Conservation Concession, MUSM 24146, 24205, 24216, 24250. Ranitomeya sirensis: MHNG 2607.18, MUSM 21116-17; Madre de Dios: Provincia Manu, Los Amigos Conservation Concession, MUSM 24199, 24217, 24227, 24232, 25861-67, 27273, 27564. Ranitomeya uakarii: Madre de Dios: Provincia Manu, Los Amigos Conservation Concession, MUSM 21068. Hemiphractidae. Gastrotheca antoniiochoai: Cusco: Provincia Paucartambo, Kosñipata, MUSM 27944-49. Gastrotheca excubitor: Cusco: Provincia Paucartambo, Kosñipata, MUSM 26277, 26280-81, KU 139193-201. Gastrotheca marsupiata: Cusco: Provincia Paucartambo, Abra Acjanaco, KU 139187-88. Gastrotheca nebulanastes: Cusco: Provincia Paucartambo, Kosñipata, CORBIDI -HE 11829, 11844, 11883-11884, 11887, MVZ 265218, MUSM 20940-44, 20949-51, 20969, 27888, 27942-43, 28060. Gastrotheca testudinea: Cusco: Provincia Paucartambo, Kosñipata, MUSM 21159-62. Hemiphractus helioi: Cusco: Provincia
Paucartambo, Kosñipata, MHNG 2606.96, MUSM 21140-41; Madre de Dios: Provincia Manu, Los Amigos Conservation Concession, MUSM 24271. Hemiphractus scutatus: Madre de Dios: Provincia Manu, Los Amigos Conservation Concession, MUSM 24252, 24315. Hylidae. Dendropsophus acreanus: Madre de Dios: Provincia Manu, Los Amigos Conservation Concession, MUSM 24276, 24284. Dendropsophus allenorum: Madre de Dios: Provincia Manu, Los Amigos Conservation Concession, MUSM 24266, 24301, 27280. Dendropsophus koechlini: Madre de Dios: Provincia Manu, Los Amigos Conservation Concession, MUSM 24285, 24289, 24311, 24323, 24332, 27278-79. Dendropsophus leali: Madre de Dios: Provincia Manu, Los Amigos Conservation Concession, MUSM 24293, 24314, 24320. Dendropsophus leucophyllatus: Cusco: Provincia Paucartambo, Kosñipata, MHNG 2607.33; Madre de Dios: Provincia Manu, Los Amigos Conservation Concession, MUSM 24178-79, 24283, 24291, 24296, 24306, 24319, 24328. Dendropsophus minutus: Cusco: Provincia Paucartambo, Kosñipata, CORBIDI -HE 11850, MHNG 2607.38-39, MUSM 21081; Madre de Dios: Provincia Manu, Los Amigos Conservation Concession, MUSM 24082-87, 24310. Dendropsophus parviceps: Cusco: Provincia Paucartambo, Kosñipata, MHNG 2607.40; Madre de Dios: Provincia Manu, Los Amigos Conservation Concession, MUSM 24240, 24292. Dendropsophus rhodopeplus: Cusco: Provincia Paucartambo, Kosñipata, MHNG 2607.41-43; Madre de Dios: 

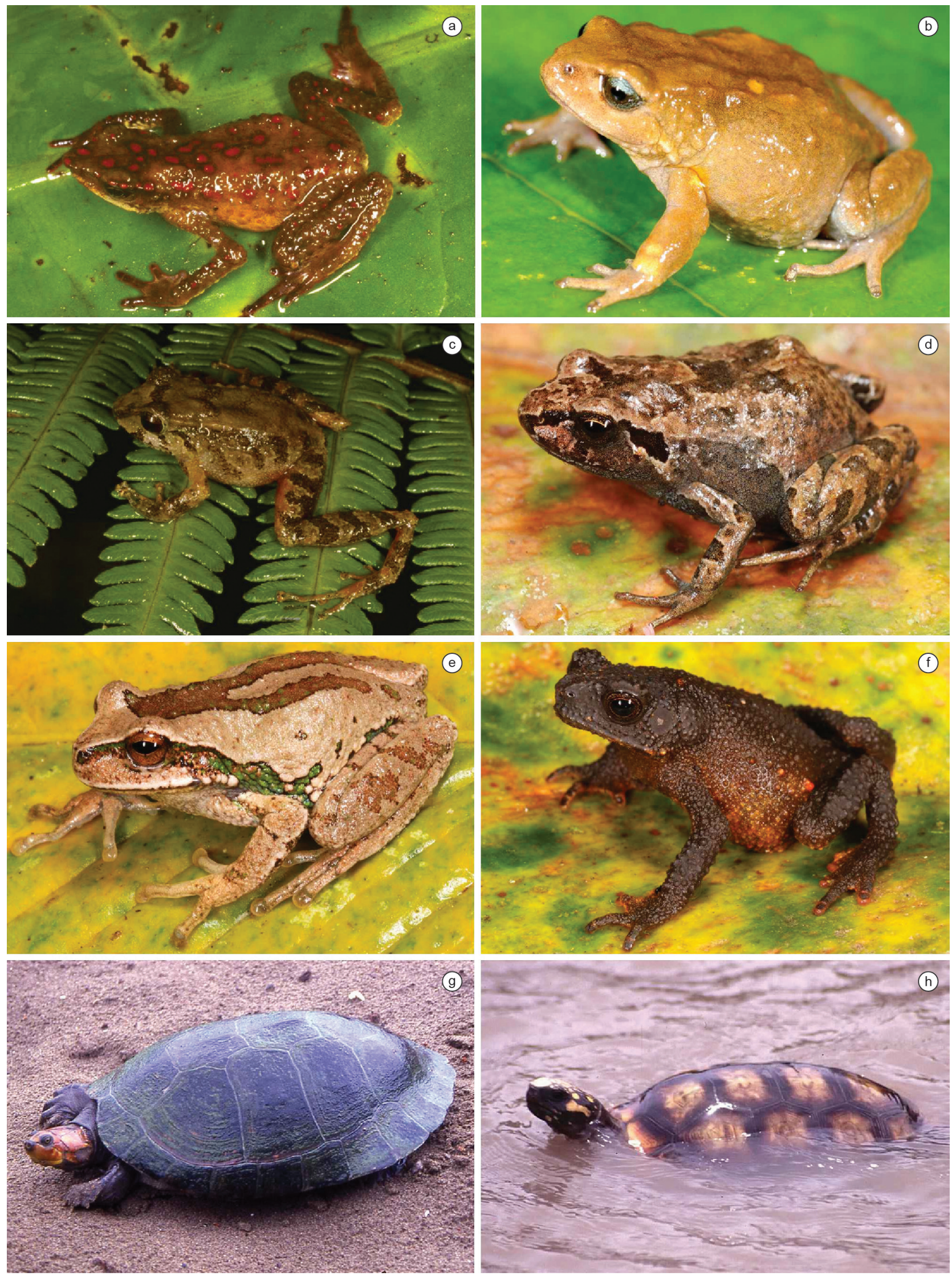

Figure 3. Threatened species of amphibians and reptiles of Manu National Park and its buffer zone. (a) Atelopus erythropus, critically endangered; (b) Bryophryne cophites, endangered; (c) Pristimantis cosnipatae, endangered; (d) Psychrophrynella usurpator, endangered; (e) Gastrotheca excubitor, vulnerable; (f) Rhinella manu, vulnerable; (g) Podocnemis unifilis, vulnerable; (h) Chelonoidis denticulata, vulnerable. Atelopus tricolor, considered vulnerable, is not included in this plate. Photos: A. Catenazzi (a-f, h), G. Knell (g). 
Provincia Manu, Los Amigos Conservation Concession, MUSM 24303. Dendropsophus sarayacuensis: Cusco: Provincia Paucartambo, Kosñipata, MHNG 2607.37. Dendropsophus schubarti: Madre de Dios: Provincia Manu, Los Amigos Conservation Concession, MUSM 24295. Dendropsophus triangulum: Madre de Dios: Provincia Manu, Los Amigos Conservation Concession, MUSM 24126, 24128, 24225. Hyloscirtus armatus: Cusco: Provincia Paucartambo, Kosñipata, MUSM 21142-48, MHNG 2606.50-53. Hyloscirtus phyllognathus: Cusco: Provincia Paucartambo, Kosñipata, MUSM 21108-10. Hyloscirtus phyllognathus: MHNG 2606.77-79. Hypsiboas boans: MHNG 2607.32. Hypsiboas calcaratus: Cusco: Provincia Paucartambo, Kosñipata, MUSM 21102; Madre de Dios: Provincia Manu, Los Amigos Conservation Concession, MUSM 24169. Hypsiboas cinerascens: Madre de Dios: Provincia Manu, Los Amigos Conservation Concession, MUSM 24249, 24313, 24324. Hypsiboas fasciatus: Cusco: Provincia Paucartambo, Kosñipata, CORBIDI -HE 11852, MHNG 2607.31; Madre de Dios: Provincia Manu, Los Amigos Conservation Concession, MUSM 24171. Hypsiboas geographicus: Cusco: Provincia Paucartambo, Kosñipata, MUSM 21158, MHNG 2607.21-23; Madre de Dios: Provincia Manu, Los Amigos Conservation Concession, MUSM 24167, 24196, 24245. Hypsiboas gladiator: Cusco: Provincia Paucartambo, Kosñipata, CORBIDI-HE 11840-42, 11848, 11872, 11881, 11905-11916, MUSM 27868-71, 27937-38, 21154-57, MHNG 2606.85-87. Hypsiboas lanciformis: Cusco: Provincia Paucartambo, Kosñipata, MUSM 21139, MHNG 2607.25-26; Madre de Dios: Provincia Manu, Los Amigos Conservation Concession, MUSM 24274, 24280. Hypsiboas punctatus: Cusco: Provincia Paucartambo, Kosñipata, CORBIDI-HE 11847, MHNG 2607.34-35; Madre de Dios: Provincia Manu, Los Amigos Conservation Concession, MUSM 24185. Osteocephalus buckleyi: Madre de Dios: Provincia Manu, Los Amigos Conservation Concession, MUSM 19225-27. Osteocephalus castaneicola: Cusco: Provincia Paucartambo, Kosñipata, MUSM 17746-49. Osteocephalus cf. leprieurii: Madre de Dios: Provincia Manu, Los Amigos Conservation Concession, MUSM 24081, 24129, 24188, 24191, 24268. Osteocephalus mimeticus: Cusco: Provincia Paucartambo, Kosñipata, CORBIDI -HE 11876, 12179, 12184, 12186, MHNG 2606.98-100, MUSM 17885-86, 17953-54, 17956, $21070-71$. Osteocephalus taurinus: Madre de Dios: Provincia Manu, Los Amigos Conservation Concession, MUSM 24248, 24258. Phyllomedusa camba: Madre de Dios: Provincia Manu, Los Amigos Conservation Concession, MUSM 24218, 24263, 24269, 24277. Phyllomedusa palliata: Madre de Dios: Provincia Manu, Los Amigos Conservation Concession, MUSM 24290, 24304. Phyllomedusa vaillanti: Cusco: Provincia Paucartambo, Kosñipata, MHNG 2607.27; Madre de Dios: Provincia Manu, Los Amigos Conservation Concession, MUSM 24309. Scarthyla goinorum: Madre de Dios: Provincia Manu, Los Amigos Conservation Concession, MUSM 24262. Scinax garbei: Cusco: Provincia Paucartambo, Kosñipata, MHNG 2607.28; Madre de Dios: Provincia Manu, Los Amigos Conservation Concession, MUSM 24281-82. Scinax ictericus: Madre de Dios: Provincia Manu, Los Amigos Conservation Concession, MUSM 24317, 24110, 24286, 24302. Scinax pedromedinae: Madre de Dios: Provincia Manu, Los Amigos Conservation Concession, MUSM 24322, 24325. Scinax ruber: Cusco: Provincia Paucartambo, Kosñipata, MUSM 21080 , MHNG 2607.14; Madre de Dios: Provincia Manu, Los Amigos Conservation Concession, MUSM 24298-99, 24330. Trachycephalus venulosus: Madre de Dios: Provincia Manu, Los Amigos Conservation Concession, MUSM 24321. Leptodactylidae. Leptodactylus andreae: Cusco: Provincia Paucartambo, Kosñipata, MHNG 2606.92, MUSM 21082; Madre de Dios: Provincia Manu, Cocha Cashu Biological Station, KU 154873, 154874, 154877-79. Leptodactylus hylaedactylus: Madre de Dios: Provincia Manu, Cocha Cashu
Biological Station, KU 154875, 154876; Madre de Dios: Provincia Manu, Los Amigos Conservation Concession, MUSM 24153, 24168, 24172, 24174, 24176-77, 24181, 24183, 24189, 24194-95, 24204, 24208, 24214, 24220, 24222, 24229, 24231, 24234, 24236-37, 24265, 24273. Leptodactylus dydimus: Madre de Dios: Provincia Manu, Los Amigos Conservation Concession, MUSM 24166, 24170, 24272. Leptodactylus griseigularis: Cusco: Provincia Paucartambo, Kosñipata, MHNG 2607.30. Leptodactylus knudseni: Madre de Dios: Provincia Manu, Los Amigos Conservation Concession, MUSM 24327. Leptodactylus leptodactyloides: Madre de Dios: Provincia Manu, Los Amigos Conservation Concession, MUSM 24193, 24256. Leptodactylus petersii: Madre de Dios: Provincia Manu, Los Amigos Conservation Concession, MUSM 24202, 24206, 24221. Leptodactylus rhodomystax: Madre de Dios: Pakitza, MVZ 19706364. Leptodactylus rhodonotus: Cusco: Provincia Paucartambo, Kosñipata, MHNG 2606.80-81, MUSM 21169-70. Leptodactylus stenodema: Madre de Dios: Provincia Manu, Los Amigos Conservation Concession, MUSM 21069. Leptodactylus wagneri: Cusco: Provincia Paucartambo, Kosñipata, MHNG 2607.29. Leiuperidae. Edalorhina perezi: Madre de Dios: Provincia Manu, Los Amigos Conservation Concession, MUSM 24242. Engystomops freibergi: Madre de Dios: Provincia Manu, Los Amigos Conservation Concession, MUSM 24247. Pleurodema marmoratum: Cusco: Provincia Paucartambo, Kosñipata, KU 173377-173379. Microhylidae. Chiasmocleis bassleri: Madre de Dios: Provincia Manu, Los Amigos Conservation Concession, MUSM 27272. Chiasmocleis ventrimaculata: Madre de Dios: Provincia Manu, Los Amigos Conservation Concession, MUSM 24209, 24215, 27260 , 27262, 27266-70. Ctenophryne geayi: Madre de Dios: Provincia Manu, Los Amigos Conservation Concession, MUSM 24200, 24294, 34318. Elachistocleis muiraquitan: Madre de Dios: Provincia Manu, Los Amigos Conservation Concession, MUSM 24224, 24278, 24329. Hamptophryne boliviana: Madre de Dios: Provincia Manu, Los Amigos Conservation Concession, MUSM 24308, 27271. Syncope antenori: Madre de Dios: Provincia Manu, Los Amigos Conservation Concession, MUSM 27263-65. Pipidae. Pipa pipa: Madre de Dios: Provincia Manu, Los Amigos Conservation Concession, MUSM 24228, 24260, 24265. Plethodonthidae. Bolitoglossa altamazonica: Madre de Dios: Provincia Manu, Los Amigos Conservation Concession, MUSM 24165, 24186, 24211, $24226,24244$. Strabomantidae. Bryophryne cophites: Cusco: Provincia Paucartambo, Kosñipata, CORBIDI -HE 11919, MHNG 2698.24, MUSM 26266-67, 26283-84, 26313, 26315, 27895-96, 30414-17, KU 138884-916. Bryophryne hanssaueri: Cusco: Provincia Paucartambo, Kosñipata, MVZ 258411-13, MHNG 2698.25, MUSM 24557, 27567-69, 27607-11, 30439-42, 30445-46, 30451, 30457, MTD 46865-66, 46887-89. Bryophryne nubilosus: Cusco: Provincia Paucartambo, Kosñipata, CORBIDI -HE 11921, MHNG 2725.27-28, MUSM 20970, 24539-40, 26310-11, 26312, 26316-17, 27882-84, 27886, 28021-22, 30413, 30422-44, 30447, 30449-50, 30455-56, MTD 47293-94. Noblella cf. myrmecoides: Cusco: Provincia Paucartambo, Kosñipata, MHNG 2606.82-84, MUSM 21072-80, 30426-29, 30458-60; Madre de Dios: Provincia Manu, Los Amigos Conservation Concession, MUSM 27261, 24219, 24251, 24266, 27274-75. Noblella pygmaea: Cusco: Provincia Paucartambo, Kosñipata, MHNG 2725.29-30, MUSM 24535-36, 26306-7, 2631820, 30423-24, 30453-54, MTD 47286-87. Noblella sp.: Cusco: Provincia Paucartambo, Kosñipata, MUSM 27581, 27582, MTD 46872-74. Oreobates cruralis: Madre de Dios: Provincia Manu, Los Amigos Conservation Concession, MUSM 24091, 24093, 24096-98, 24100, 24102-04, 24107. Oreobates gemcare: Cusco: Provincia Paucartambo, Kosñipata, CORBIDI -HE 11922, MHNG 2606.46-47, MUSM 21086, 21151, 27885, 27901, 27903-4, 28029, 21152-53, 
MTD 46893. Oreobates granulosus: Cusco: Provincia Paucartambo, Kosñipata, MUSM 30554-55. Oreobates quixensis: Cusco: Provincia Paucartambo, Kosñipata, MHNG 2607.36; Madre de Dios: Provincia Manu, Los Amigos Conservation Concession, MUSM 24305. Pristimantis altamazonicus: Cusco: Provincia Paucartambo, Kosñipata, CORBIDI -HE 11854; Madre de Dios: Provincia Manu, Los Amigos Conservation Concession, MUSM 24108, 25848, 25858. Pristimantis carvalhoi: Madre de Dios: Provincia Manu, Los Amigos Conservation Concession, MUSM 24088, 24095, 24105-06. Pristimantis cosnipatae: Cusco: Provincia Paucartambo, Kosñipata, MHNG 2606.72, MUSM 20994-95, 21094, 27890, KU 138876, 162298-304. Pristimantis danae: Cusco: Provincia Paucartambo, Kosñipata, CORBIDI -HE 11823, 11828, 11831, 11836, 11851, 11857, 11859-61, 11873, 11879, 11882, 11885, 11897, 11904, 11918, 11923, 12176-77, MHNG 2606.88-91, MUSM 20996-97, 21163-68, 26268, 26275, 27897-27900, 28023, 28030, 30419-20, KU 16230750. Pristimantis divnae: Madre de Dios: Provincia Manu, Los Amigos Conservation Concession, MUSM 19990, 27276-77, 27281. Pristimantis fenestratus: Cusco: Provincia Paucartambo, Kosñipata, MHNG 2607.24, MUSM 21118; Madre de Dios: Provincia Manu, Los Amigos Conservation Concession, MUSM 24092, 24094. Pristimantis lindae: Cusco: Provincia Paucartambo, Kosñipata, CORBIDI -HE 11837, 11866, 11888, MHNG 2606.97, MUSM 20998, 21112-13, 27902, 27905, 27907-8, 28020, 30448, 30470, KU KU 162305. Pristimantis mendax: Cusco: Provincia Paucartambo, Kosñipata, CORBIDI -HE 11862, MHNG 2607.11-13, MUSM 20999, 21105-7. Pristimantis mercedeae: Cusco: Provincia Paucartambo, Kosñipata, MUSM 21095. Pristimantis ockendeni: Madre de Dios: Provincia Manu, Los Amigos Conservation Concession, MUSM 24089, 24099, 24109. Pristimantis pharangobates: Cusco: Provincia Paucartambo, Kosñipata, CORBIDI -HE 11822, 11833, 11846, 11856, 11875, 11880, 11886, 11899 11901, 11903, 11920, 12178, MHNG 2606.42-45, 2606.73-76, MUSM 17975, 26265, 26269-71, 26276, 27622-28, 27909-12, 28031, 30418, 30433, MTD 47483-87, KU 138877. Pristimantis platydactylus: Cusco: Provincia Paucartambo, Kosñipata, CORBIDI -HE 11835, 11838, 11843, 11845, 11849, 11858, 11863-65, 1186970, 11874, 11894-95, 11902, 12180-81, 12189-90, MHNG 2606.64, 2606.66-67, 2606.71, MUSM 21088-90, 21096, 21100, 26286-92, 26305, 27914-15, 27917, 28048, 30432, 30461-63, KU 162306. Pristimantis reichlei: Cusco: Provincia Paucartambo, Kosñipata, CORBIDI -HE 11853, 11855, MHNG 2607.15-17, MUSM 21103; Madre de Dios: Provincia Manu, Los Amigos Conservation Concession, MUSM 24101. Pristimantis salaputium: Cusco: Provincia Paucartambo, Kosñipata, CORBIDI -HE 11827, 12182, 12185, 12187-88, MHNG 2606.65, MUSM 21087, 21091-93, $21097-$ 98, 27913, 27916, 27918, 27920, 30452, 30464-67, 30469, KU 162291-97. Pristimantis tantanti Madre de Dios: Cocha Cashu Field Station: MUSM 3848, 9119. Pristimantis toftae: Cusco: Provincia Paucartambo, Kosñipata, CORBIDI -HE 11832, 11871, 11877-78, 11889-93, 11898, MHNG 2607.01-03, MUSM 21003, 21119-21, 27921-22, 27924-28; Madre de Dios: Provincia Manu, Los Amigos Conservation Concession, MUSM 24090. Pristimantis ventrimarmoratus: Cusco: Provincia Paucartambo, Kosñipata, MUSM 21101. Psychrophrynella usurpator: Cusco: Provincia Paucartambo, Kosñipata, MUSM 20011, 20873-81, 20896-20913, 20925-33, 20946-47, 20955-57, 21012-18, 26272-73, 26278-79, 26308, 27592, 27906, 27950, 28033-28047, 30303, 30305, 3039630400, 30405-30409, 30471-30474, MTD 46881-82, KU 138917. 138919-78. Psychrophrynella sp. P: Cusco: Provincia Paucartambo, Kosñipata, MHNG 2606.56, MUSM 20958-60, 20963, 20973-74, 20977, 21186-87, 26274, 26300-4, 26314, 27586-91, 27594-27604, 27936, 30395, 30410-11, 30425, 30475, 30478-80, 20945, 20961-62,
20964, 20975-76, MTD 46876-80, 46883, 46885-86. Psychrophrynella sp. R: Cusco: Provincia Paucartambo, Kosñipata, MUSM 26294-99, 27584-85, 27593, 27605, 27891, 30421, 30476-77, MTD 46875, 46884.

REPTILES. Anguidae. Diploglossus fasciatus: Madre de Dios: Pakitza, MVZ 197116. Boidae. Corallus hortulanus: Madre de Dios: Pakitza, MVZ 197158-59. Colubridae. Clelia clelia: Madre de Dios: Pakitza, MVZ 197163. Chironius carinatus: Madre de Dios: Provincia Manu, Los Amigos Conservation Concession, MUSM 24073. Chironius fuscus: Madre de Dios: Provincia Manu, Los Amigos Conservation Concession, MUSM 24353, 24365. Dendrophidion dendrophis: Madre de Dios: Provincia Manu, Los Amigos Conservation Concession, MUSM 24349, 24352. Dipsas catesbyi: Madre de Dios: Provincia Manu, Los Amigos Conservation Concession, MUSM 24343. Dipsas peruana: Cusco: Provincia Paucartambo, Kosñipata, CORBIDI -HE 11839. Drepanoides anomalus: Madre de Dios: Pakitza, MVZ 197146. Drymoluber dichrous: Madre de Dios: Provincia Manu, Los Amigos Conservation Concession, MUSM 24362. Helicops angulatus: Madre de Dios: Provincia Manu, Los Amigos Conservation Concession, MUSM 24113, 24139, 24340, 24358. Imantodes cenchoa: Madre de Dios: Provincia Manu, Los Amigos Conservation Concession, MUSM 24110, 24348. Leptodeira annulata: Madre de Dios: Provincia Manu, Los Amigos Conservation Concession, MUSM 24364, 24369. Leptophis ahaetulla: Madre de Dios: Provincia Manu, Los Amigos Conservation Concession, MUSM 24357, 24367. Liophis reginae: Madre de Dios: Provincia Manu, Los Amigos Conservation Concession, MUSM 24339. Liophis taeniogaster: Madre de Dios: Provincia Manu, Los Amigos Conservation Concession, MUSM 24363, 34368. Liophis taeniurus: Cusco: Provincia Paucartambo, Kosñipata, CORBIDI -HE 11896. Oxyrhopus marcapatae: Cusco: Provincia Paucartambo, Kosñipata, MUSM 30251-52. Oxyrhopus melanogenys: Madre de Dios: Pakitza, MVZ 197150. Pseudoboa coronata: Madre de Dios: Pakitza, MVZ 1974148. Pseudoeryx plicatilis: Madre de Dios: Provincia Manu, Los Amigos Conservation Concession, MUSM 24359. Siphlophis compressus: Madre de Dios: Provincia Manu, Los Amigos Conservation Concession, MUSM 24338; Pakitza, MVZ 197145. Tachymenis peruviana: MUSM 28014, KU 139426-27. Taeniophallus brevirostris: Madre de Dios: Provincia Manu, Los Amigos Conservation Concession, MUSM 25860. Taeniophallus occipitalis: Madre de Dios: Provincia Manu, Los Amigos Conservation Concession, MUSM 25859. Xenopholis scalaris: Madre de Dios: Provincia Manu, Los Amigos Conservation Concession, MUSM 24162, 24350. Gekkonidae. Gonatodes humeralis: Madre de Dios: Provincia Manu, Los Amigos Conservation Concession, MUSM 24120, 24142-43, 24161. Pseudogonatodes guianensis: Madre de Dios: Provincia Manu, Los Amigos Conservation Concession, MUSM 24137, 24150, 24152, 24154, 24230, 24257. Elapidae. Micrurus annellatus: Cusco: Provincia Paucartambo, Kosñipata, MUSM 28016. Micrurus lemniscatus: Madre de Dios: Provincia Manu, Los Amigos Conservation Concession, MUSM 24111. Micrurus obscurus: Madre de Dios: Provincia Manu, Los Amigos Conservation Concession, MUSM 24341. Gymnophthalmidae. Bachia dorbignyi: Madre de Dios: Provincia Manu, Los Amigos Conservation Concession, MUSM 24151. Cercosaura eigenmanni: Madre de Dios: Provincia Manu, Los Amigos Conservation Concession, MUSM 24121, 24148, 24156-58. Potamites sp.: Cusco: Provincia Paucartambo, Kosñipata, MUSM 26309, 26321, 28055-59, 30258-62, 30305. Proctoporus bolivianus: Cusco: Provincia Paucartambo, Kosñipata, CORBIDI -HE 11830, MUSM 27617, 30263-90, 30296, MTD 46894, 47479. Ptychoglossus brevifrontalis: Madre de Dios: Provincia Manu, Los Amigos Conservation Concession, MUSM 24117-18, 24122, 24133, 
24138, 24145. Leptotyphlopidae. Leptotyphlops diaplocius: Madre de Dios: Provincia Manu, Los Amigos Conservation Concession, MUSM 24147, 24159. Polychrotidae. Anolis cuscoensis: Cusco: Provincia Paucartambo, Kosñipata, CORBIDI -HE 11825-26, MUSM 30255-57. Anolis fuscoauratus: Madre de Dios: Provincia Manu, Los Amigos Conservation Concession, MUSM 24116, 24119, 24132, 24134, 24144, 24149, 24160. Anolis nitens: Madre de Dios: Provincia Manu, Los Amigos Conservation Concession,MUSM 24114, 24136. Anolis ortonii: Madre de Dios: Provincia Manu, Los Amigos Conservation Concession, MUSM 24155. Scincidae. Copeoglossum nigropunctatum: Madre de Dios: Provincia Manu, Los Amigos Conservation Concession,MUSM 24141. Teiidae. Ameiva ameiva: Madre de Dios: Provincia Manu, Los Amigos Conservation Concession, MUSM 24140. Kentropyx pelviceps: Madre de Dios: Provincia Manu, Los Amigos Conservation Concession, MUSM 24112. Tupinambis teguixin: Cusco: Provincia Paucartambo, Kosñipata, KU 139420. Typhlopidae. Typhlops reticulatus: Madre de Dios: Provincia Manu, Los Amigos Conservation Concession, MUSM 24261. Viperidae. Bothrops andianus: Cusco: Provincia Paucartambo, Kosñipata, CORBIDI -HE 11834, MUSM 30297. Bothrops atrox: Madre de Dios: Provincia Manu, Los Amigos Conservation Concession, MUSM 24336, 24356. Bothriopsis bilineata: Madre de Dios: Provincia Manu, Los Amigos Conservation Concession, MUSM 24366. Bothrops brazili: Madre de Dios: Provincia Manu, Los Amigos Conservation Concession, MUSM 24074.

\section{Acknowledgments}

We thank the administration of Manu NP, Cusco for issuing research permit and for supporting our work in the park and its buffer zone. We thank two anonymous reviewers for comments. Collecting and export permits were issued by the Instituto Nacional de Recursos Naturales and the Dirección de Gestión Forestal y de Fauna Silvestre at the Ministry of Agriculture in Lima (research permit numbers: 0682-96-AG, 0346-98-AG, 89-98-INRENA-DGANPFS-DANP, 008-2002-INRENA-J-DGFFS-DCB, 012-2003-INRENA-IFFSDCB, 053-2005-INRENA-IFFS-DCB, 23-2006-INRENA-IFFSDCB, 044-C/C-2006-INRENA-IANP, 67-2007- INRENA-IFFSDCB, 11-2008-INRENA-IFFS-DCB) and Manu National Park in Cusco (06-2013-SERNANP-PNM-JEF). We thank park rangers, students, and colleagues who assisted in field work. We thank the following colleagues for verifying some of the species identifications: Ronald Heyer, Lily Rodriguez, Claudia Torres, Marvalee Wake, Hussan Zaher. RvM was supported by a National Science Foundation Postdoctoral Research Fellowship in Biology (DBI-1103087). Fieldwork was supported by grants from the Amazon Conservation Association (AC, RvM), the Rufford Small Grants Foundation (AC), the Chicago Board of Trade Endangered Species Fund (AC), the Amphibian Specialist Group (AC and EL), the Fondation MatheyDupraz (AC), the Swiss Academy of Natural Sciences (AC), the Wildlife Conservation Society (RvM), and the National Geographic Society Committee for Research and Exploration (Grant \# 9191-12 to RvM). This is publication No. 2 of the Amphibian Specialist Group Peru network.

\section{References}

BLACKBURN, D.C. \& WAKE, D.B. 2011. Class Amphibia Gray, 182. In Animal biodiversity: An outline of higher-level classification and survey of taxonomic richness (Z.-Q. Zhang, Ed.). Zootaxa. 3148:39-55.

CAMPBELL, J.A. \& LAMAR, W.W. 1989. The venomous reptiles of Latin America. Comstock Publishing Associates, Ithaca, New York.
CASTOE, T.A., DOAN, T.M. \& PARKINSON, C.L. 2004. Data partitions and complex models in Bayesian analysis: the phylogeny of Gymnophthalmid lizards. System. Biol. 53(3):448-469. PMid:15503673. http://dx.doi. org $/ 10.1080 / 10635150490445797$

CASTROVIEJO-FISHER, S., PADIAL, J.M., CHAPARRO, J.C., AGUAYO, R. \& DE LA RIVA, I. 2009. Anew species of Hyalinobatrachium (Anura: Centrolenidae) from the Amazonian slopes of the central Andes, with comments on the diversity of the genus in the area. Zootaxa 2143:24-44.

CATENAZZI, A. \& RODRÍGUEZ, L.O. 2001. Diversidad, distribución y abundancia de anuros en la parte alta de la Reserva de Biosfera del Manu. In El Manu y otras experiencias de investigación y manejo de bosques neotropicales. (L.O. Rodriguez, ed.). Pro-Manu, Cusco, p.53-57.

CATENAZZI, A., LEHR, E., RODRIGUEZ, L.O. \& VREDENBURG, V. T. 2011. Batrachochytrium dendrobatidis and the collapse of anuran species richness and abundance in the upper Manu National Park, Peru. Cons. Biol. 25(2):382-391. PMid:21054530.

CATENAZZI, A., RODRÍGUEZ, L. \& DONNELLY, M.A. 2009. The advertisement calls of four species of glassfrogs (Centrolenidae) from Southeastern Peru. Studies Neotrop. Fauna Environ. 44(2):83-91. http:// dx.doi.org/10.1080/01650520903036653

CATENAZZI, A., VON MAY, R., LEHR, E., GAGLIARDI-URRUTIA, G. \& GUAYASAMIN, J. M. 2012. A new, high-elevation glassfrog (Anura: Centrolenidae) from Manu National Park, southern Peru. Zootaxa 3388:56-68.

CHAPARRO, J.C., PRAMUK, J.B. \& GLUESENKAMP, A.G. 2007. A new species of arboreal Rhinella (Anura: Bufonidae) from could forest of southeastern Peru. Herpetologica 63(2):203-212. http://dx.doi. org/10.1655/0018-0831(2007)63[203:ANSOAR]2.0.CO;2

DE LA RIVA, I., \& CHAPARRO, J. C. 2005. A new species of tree frog from the Andes of southeastern Peru (Anura: Hylidae: Hyla).AmphibiaReptilia, 26(4): 515-521.

DE LA RIVA, I., CHAPARRO, J.C. \& PADIAL, J.M. 2008. A new, longstanding misidentified species of Psychrophrynella Hedges, Duellman \& Heinicke from Departamento Cusco, Peru (Anura: Strabomantidae). Zootaxa. 1823:42-50.

DUELLMAN, W.E. 1976. Centrolenid frogs from Peru. Occas. Papers Mus. Nat. Hist., Univ. Kansas 52:1-11.

DUELLMAN, W.E. 1978. New species of Leptodactylid frogs of the genus Eleutherodactylus from the Cosñipata Valleu, Perú. Proc.Biol. Soc. Washington 91:418-430.

DUELLMAN, W.E. 1999. Distribution patterns of amphibians in South America. In Patterns of distribution of amphibians: A global perspective (Duellman, W.E., ed.). The John Hopkins University Press, Baltimore and London, p.255-328.

DUELLMAN, W.E. 2005. Cusco Amazónico: The lives of amphibians and reptiles in an Amazonian rainforest. Comstock Publishing Associates, Ithaca, New York. PMid:16403648.

DUELLMAN, W.E. \& LEHR, E. 2009. Terrestrial-Breeding frogs (Strabomantidae) in Peru. Natur und Tier-Verlag, Münster, 384 p.

DUELLMAN, W.E., CATENAZZI, A. \& BLACKBURN, D.C. 2011. A new species of marsupial frog (Anura:Hemiphractidae: Gastrotheca) from the Andes of southern Peru. Zootaxa 3095:1-14.

FROST, D.R. 2013. Amphibian Species of the World: an Online Reference. Version 5.6 (January 2013). http://research.amnh.org/vz/herpetology/ amphibia (último accesso em 6/10/2013).

GRISCOM, B.W., DALY, D.C. \& ASHTON, M.S. 2007. Floristics of bamboo-dominated stands in lowland terra-firma forests of southwestern Amazonia. J. Torrey Bot. Soc. 134:108-125. http://dx.doi. org/10.3159/1095-5674(2007)134[108:FOBSIL]2.0.CO;2

GUTBERLET, R.L. \& CAMPELL, J. A. 2001. Generic recognition for a neglected lineage of South American Pitvipers (Squamata: Crotalinae) with the description of a new species from the Colombian Chocóo. American Museum Novitates 3316: 1-15.

HARVEY, M.B. 1999. Revision of Bolivian Apostolepis (Squamata: Colubridae). Copeia 1999(2):388-409. http://dx.doi.org/10.2307/1447485 
HURTADO, J.L. \& BLANCO, D. 1994. Nuevo registro de ofidios del bosque nublado del valle de Q'ósñipata, Cusco. Bol. Lima 91-96:49-52.

LARSEN, T.H., LOPERA, A. \& FORSYTH, A. 2006. Extreme trophic and habitat specialization by Peruvian dung beetles (Coleoptera: Scarabaeidae: Scarabaeinae). Coleopt. Bull. 60(4):315-324. http://dx.doi. org/10.1649/0010-065X(2006)60[315:ETAHSB]2.0.CO;2

LE, M., RAXWORTHY, C.J., McCORD, W.P. \& MERTZ, L. 2006. A molecular phylogeny of tortoises (Testudines: Testudinidae) based on mitochondrial and nuclear genes. Molec. Phylogen. Evol. 40(2):517-531. PMid:16678445. http://dx.doi.org/10.1016/j.ympev.2006.03.003

LEHR, E. \& CATENAZZI, A. 2008. A new species of Bryophryne (Strabomantidae) from southern Peru. Zootaxa 1784:1-10.

LEHR, E. \& CATENAZZI, A. 2009a. A new species of minute Noblella (Anura: Strabomantidae) from southern the smallest frog of the Andes. Copeia 2009(1):148-156. http://dx.doi.org/10.1643/CH-07-270

LEHR, E. \& CATENAZZI, A. 2009b. Three new species of Bryophryne from southern Peru. S. Amer. J. Herpet. 4(2):125-138. http://dx.doi. org/10.2994/057.004.0204

LEHR, E. \& VON MAY, R. 2009. A new species of Pristimantis (Anura: Strabomantidae) from the Amazonian lowlands of southern Peru. J. Herpet. 43(3):485-494. http://dx.doi.org/10.1670/08-202R.1

McCORD, W.P., MEHDI, J.-O. \& LAMAR, W.W. 2001. A taxonomic reevaluation of Phrynops (Testudines: Chelidae) with the description of two new genera and a new species of Batrachemys. Rev. Biol. Trop. 49(2):715-764. PMid:11935927.

MORALES, V.R. \& McDIARMID, R.W. 1996. Annotated checklist of the amphibians and reptiles of Pakitza, Manu National Park Reserve Zone, with comments on the herpetofauna of Madre de Dios, Peru. In Manu: The biodiversity of southeastern Peru (Wilson, D.E. \& A. Sandoval, eds.). Smithsonian Institution, Washington, p.503-522.

MYERS, N., MITTERMEIER, R.A., MITTERMEIER, C.G., DA FONSECA, G.A.B. \& KENT, J. 2000. Biodiversity hotspots for conservation priorities. Nature 403:853-858. PMid:10706275. http://dx.doi. org $/ 10.1038 / 35002501$

NAVAS, C.A. 2003. Herpetological diversity along Andean elevational gradients: links with physiological ecology and evolutionary physiology. Comp. Biochem. Physiol. A 133(3):469-485. http://dx.doi.org/10.1016/ S1095-6433(02)00207-6

PADIAL, J.M., CHAPARRO, J.C. \& DE LA RIVA, I. 2007. A new species of the Eleutherodactylus discoidalis group (Anura: Brachycephalidae) from cloud forests of Peru. Herpetologica 63(1):114-122. http://dx.doi. org/10.1655/0018-0831(2007)63[114:ANSOTE]2.0.CO;2

PADIAL, J.M., CHAPARRO, J.C.; KÖHLER, J. \& DE LA RIVA, I. 2009. Rediscovery, resurrection and redescription of Rhinella leptoscelis (Boulenger, 1912) (Anura: Bufonidae). Zootaxa. 2115:56-64.

PADIAL, J.M., CHAPARRO, J.C., CASTROVIEJO-FISHER, S., GUAYASAMIN, J. M., LEHR, E., DELGADO, A.J., VAIRA, M., TEIXEIRA, JUNIOR, M., AGUAYO-VEDIA, C.R. \& DE LA RIVA, I. 2012. A revision of species diversity in the Neotropical genus Oreobates (Anura: Strabomantidae), with the description of three new species from the Amazonian slopes of the Andes. Amer. Mus. Novitates. 3752:1-55. http://dx.doi.org/10.1206/3752.2
PITMAN, N.C.A., TERBORGH, J., SILMAN, M.R., \& NUÑEZ, P. 1999. Tree species distributions in an upper Amazonian forest. Ecology 80(8):26512661. http://dx.doi.org/10.1890/0012-9658(1999)080[2651:TSDIAU] 2.0.CO;2

RODRÍGUEZ, L. 1987. Parque Nacional del Manu: Lista preliminar de los batracios anuros de Cocha Cashu. Bol. Lima 53:88-90.

RODRÍGUEZ, L.O. 1992. Structure et organisation du peuplement d'anoures de Cocha Cashu, Parque National Manu, Amazonie Péruvienne. Terre et Vie 47(2):151-197.

RODRÍGUEZ, L.B. \& CADLE, J.E. 1990. A preliminary overview of the herpetofauna of Cocha Cashu, Manu National Park, Peru. In Four Neotropical Rainforests (A.H., Gentry, ed.). Yale University Press, New Haven, p.410-425.

SEIMON, T.A., SEIMON, A., DASZAK, P., HALLOY, S., SCHLOEGEL, L., AGUILAR, C., SOWELL, P., HYATT, A., KONECKY, B. \& SIMMONS, J.E. 2007. Upward range extension of Andean anuran and chytridiomycosis to extreme elevations in response to tropical deglaciation. Glob. Change Biol. 13(1):288-299. http://dx.doi.org/10.1111/j.1365-2486.2006.01278.x

SERNANP. 2010. Áreas naturals protegidas. Sernanp, Frononanpe, Lima.

STUART, S.N., CHANSON, J.S., COX, N.A., YOUNG, B.E., RODRIGUES, A.S.L., FISCHMAN, D.L. \& WALLER, R.W. 2004. Status and trends of amphibian declines and extinction worldwide. Science 306:1783-1786. PMid:15486254. http://dx.doi.org/10.1126/science.1103538

UETZ, P. \& HOŠEK, J. 2013. The Reptile Database. http://www.reptiledatabase.org (último acesso em 6/10/2013).

VON MAY, R., CATENAZZI, A., ANGULO, A., BROWN, J.L., CARRILLO, J., CHÁVEZ, G., CÓRDOVA, J.H., CURO, A., DELGADO, A., ENCISO, M.A., GUTIÉRREZ, R., LEHR, E., MARTÍNEZ, J.L., MEDINAMÜLLER, M., MIRANDA, A., NEIRA, D.R., OCHOA, J.A., QUIROZ, A.J., RODRÍGUEZ, D.A., RODRIGUEZ, L.O., SALAS, A.W., SEIMON, T., SEIMON, A., SIU-TING, K., SUÁREZ, J., TORRES, C. \& TWOMEY, E. 2008. Current state of conservation knowledge on threatened amphibian species in Peru. Trop. Cons. Sci. 1(4):376-396.

VON MAY, R., EMMONS, L.H., KNELL, G., JACOBS, J.M. \& RODRÍGUEZ, L.O. 2006. Reptiles del Centro Río Los Amigos, Manu y Tambopata, Perú. Rapid Color Guide \#194. http://fm2.fieldmuseum. org/plantguides/guideimages.asp?ID=328.

VON MAY, R. \& DONNELLY, M.A 2009. Do trails affect relative abundance estimates of rainforest frogs and lizards?. Aust. Ecol. 34(6):613-620.

VON MAY, R., SIU-TING, K., JACOBS, J.M., MEDINA-MÜLLER, M., GAGLIARDI, G., RODRÍGUEZ, L.O. \& DONNELLY, M.A. 2009. Species diversity and conservation status of amphibians in Madre de Dios, Perú. Herpet. Cons. Biol. 4(1):14-29.

VON MAY, R. 2009. Diversity patterns of amphibians in lowland amazonian forests in southeastern Peru. PhD dissertation, Florida International University, Miami, 201p.

VON MAY, R., JACOBS, J.M., SANTA CRUZ, R., VALDIVIA, J., HUAMÁN, J. \& DONNELLY, M.A. 2010. Amphibian community structure as a function of forest type in Amazonian Peru. J. Trop. Ecol. 26(5):509-519. http://dx.doi.org/10.1017/S0266467410000301 